\title{
¿QUÉ SE LEE EN LOS ESTUDIOS DOCTORALES?: ESTUDIO EMPÍRICO BASADO EN GÉNEROS A TRAVÉS DEL DISCURSO ACADÉMICO DE SEIS DISCIPLINAS*
}

\author{
WHAT DO STUDENTS READ IN DOCTORAL STUDIES?: \\ EMPIRICAL RESEARCH BASED ON GENRES ACROSS THE \\ ACADEMIC DISCOURSE OF SIX DISCIPLINES
}

\author{
GIOVANNI PARODI ${ }^{* *}$ \\ Pontificia Universidad Católica de Valparaíso. Valparaíso, Chile. \\ gparodi@ucv.cl
}

\section{RESUMEN}

En este artículo nos interesa conocer los mecanismos discursivos escritos por medio de los cuales los estudiantes doctorales acceden a la información especializada y se posibilita así la construcción del conocimiento a través de diversas disciplinas. Así, los objetivos de este artículo son identificar y describir los géneros del discurso académico a partir de un corpus de 3.147 textos, recolectados en seis disciplinas de las Ciencias Sociales y Humanidades y de las Ciencias Básicas (Corpus PUCV-2010). Una vez identificados los géneros, se cuantifica su ocurrencia en términos generales así como por cada una de las seis disciplinas en estudio: Biotecnología, Física, Historia, Lingüística, Literatura y Química. Los principales hallazgos muestran la identificación de 30 géneros. Entre ellos, los de mayor ocurrencia son el Texto Disciplinar, el Artículo de Investigación y el Manual. El discurso académico de la Literatura cuenta con la mayor diversidad de géneros (15), mientras que el discurso académico de la Química y de la Física son en los que se identifica menor variación genérica (5).

Palabras clave: Género del discurso, discurso académico, Corpus PUCV-2010, disciplinaridad, estudios de doctorado.

* Investigación financiada por el Proyecto FONDECYT 1090030.

** La investigación aquí reportada no habría podido llegar a materializarse en esta publicación sin el compromiso, trabajo sostenido y notable empeño que han demostrado los integrantes del grupo de investigación FONDECYT 1090030, durante los 4 años de este proyecto. Por todo ello, agradezco a Romualdo Ibáńez, René Venegas, Carolina Aguilera, Carolina Flores, Jadranka Gladic, Cristóbal Julio, Enrique Boudon, Andrea Santana, Betsabé Delgado, Marco Molina, Waldo Carvallo, Cristián Aravena, Ariel Mendoza, Alejandro Córdova y Gilda Bastías. Por supuesto, los contenidos e interpretaciones aquí expuestas son de exclusiva responsabilidad del autor. 


\section{ABSTRACT}

In this study we are concerned with describing written discourse tools employed by university $\mathrm{PhD}$ students to access specialized information and to construct knowledge across disciplines. In this vein, the research objectives are to identify and to describe the academic discourse genres based on a corpus of 3,147 texts collected in six disciplines in Social Sciences and Humanities and Basic Sciences (PUCV-2010 Corpus). Once the genres are identified, their occurrence is quantified across the disciplines under study: Biotechnology, Chemistry, History, Linguistics, Literature, and Physics. Among the most important findings, 30 genres were identified. The most recurrent genres are: Disciplinary Text, Research Article, and Textbook. Literature academic discourse shows the principal genre variation (15), while Chemistry and Physics academic discourse reveals the least genre variation (5).

Keywords: Discourse genres, academic discourse, PUCV-2010 Corpus, disciplinarity, PhD studies.

Recibido: 07.09.2012. Aceptado: 05.10.2012.

\section{INTRODUCCIÓN}

T as investigaciones contemporáneas en el marco de la Lingüística de Corpus, el Análisis del Género y el Discurso Disciplinar han aportado hallazgos contundentes acerca de los textos que circulan en contextos académicos y profesionales y de las variaciones genéricas prototípicas en el discurso de diversas disciplinas (Parodi, 2008a, 2010a, 2012b; Farlora, 2011; Araya, 2011; Burdiles, 2012; Jarpa, 2012; Martínez, 2012). Estos datos empíricos están aportando descripciones significativas para los procesos de alfabetización tanto académica como profesional e impulsan programas de instrucción para el desarrollo de las habilidades de comprensión y producción de textos de diversa naturaleza en español como lengua materna (Castañeda y Henao, 1995; Peronard, Gómez, Parodi, Núñez y González, 1998; Marinkovich, Peronard y Parodi, 2006; Arnoux, 2002, 2009; Marinkovich, Velásquez, Salazar y Córdova, 2009; Resnik y Valente, 2009; Parodi, 2010a, 2012; Cubo de Severino, Lacon y Puiatti, 2012; PLEA, 2012; entre otros).

No obstante estos avances y desarrollos, aún es incipiente la descripción de los géneros que se leen y escriben en contextos académicos y profesionales a través de las disciplinas; sobre todo, se hace necesario contar con estudios a partir de investigaciones basadas en corpus de textos amplios, ecológicos y diversificados.

Si bien en los últimos años hemos estado interesados en la identificación de géneros especializados a nivel de la educación técnico-profesional (Parodi, 2005) y de la educación superior a nivel de pregrado (Parodi, 2008a; Ibáñez, 2008; Arave- 
na, 2010; González, 2011; Grassi, 2012; Castillo, 2012; Faune, 2012; Rodríguez, 2012; Zamora, 2012), sólo recientemente nos hemos enfocado en los contextos de postgrado, tanto en magíster como doctorado (Parodi, 2010b; Martínez, 2012; Lobos, 2012). Nuestro interés actual dice relación con conocer los mecanismos discursivos escritos por medio de los cuales los alumnos doctorales acceden a la información especializada y se posibilita así la construcción del conocimiento a través de diversas disciplinas. En este marco, los objetivos de este artículo, en el contexto de un proyecto de mayor envergadura, son identificar y describir los géneros del discurso académico a partir de un corpus de 3.147 textos, recolectados en seis disciplinas de las Ciencias Sociales y Humanidades y de las Ciencias Básicas (Corpus PUCV-2010). Para ello, se identifican los géneros a partir de un estudio de los textos del corpus y se cuantifica su ocurrencia en términos generales así como por cada una de las seis disciplinas en estudio: Biotecnología, Física, Historia, Lingüística, Literatura y Química.

\section{MARCO DE REFERENCIA}

Como se sabe, comparativamente ha existido mayor desarrollo de estudios aplicados, centrados en la escritura a través de las disciplinas, que de la lectura en las especialidades. En efecto, es fácil comprobar el tremendo auge primero desde la lengua inglesa, tanto materna como extranjera y, más recientemente, en español como lengua materna de estudios descriptivos acerca de géneros disciplinares y de la creación y puesta en marcha de programas de escritura de géneros especializados, tanto académicos como profesionales. Por el contrario, es muy incipiente y comparativamente más escasa la investigación acerca de los procesos de comprensión de textos escritos especializados y de estudios contrastivos en esta habilidad psicolingüística. Podría esto ser similar al progreso dispar que existió en el desarrollo de teorías y modelos de lectura y escritura durante la segunda mitad del siglo XX, tal como constata la indagación realizada por Parodi (2003). En ese estudio, Parodi declaraba que la investigación en comprensión desde los marcos contemporáneos había -hasta ese momento- recibido mayor atención y auge, en cuanto a desarrollo de teorías y modelos, en comparación con la de la escritura.

Esta podría ser ciertamente una paradoja. Si bien durante la primera mitad del siglo veinte habría existido un relativo mayor desarrollo de teorías y modelos de comprensión de textos escritos, en oposición a los de escritura (Parodi, 2003), durante los comienzos del actual siglo son la escritura en las disciplinas y a través del currículo, áreas que muestran avances tremendos (Marinkovich, Morán y Benítez, 1997; Bazerman y Wiener, 2009; Arnoux, 2009; Bazerman, Krut, Lunsford, McLeod, Null, Rogers y Stansell, 2009; Bazerman, Dean, Early, Lunsford, Null, Rogers y Stansell, 2012; Bazerman, 1994, 2012; Marinkovich, 2012), en 
comparación con la lectura en las disciplinas y el currículo. Este auge en escritura estaría marcado por los movimientos emergentes desde la Teoría del Género (Swales, 1990, 2004; Bhatia, 1993, 2004), la Escritura a través del Currículo (Miller, 1984; Freedman y Medway, 1994; Bazerman y Russell, 1995) y la Escritura en las Disciplinas (Russell, 2002).

Desde este escenario, nuestra investigación se sitúa en el contexto de los géneros académicos universitarios. Nos interesan aquellos géneros que circulan en el sistema terciario en su nivel máximo de educación superior, esto es, el de doctorado. Tal como se muestra en la Figura 1, entendemos que existe una amplia variedad de géneros a través de los cuales un lector recorre su formación académica y profesional.

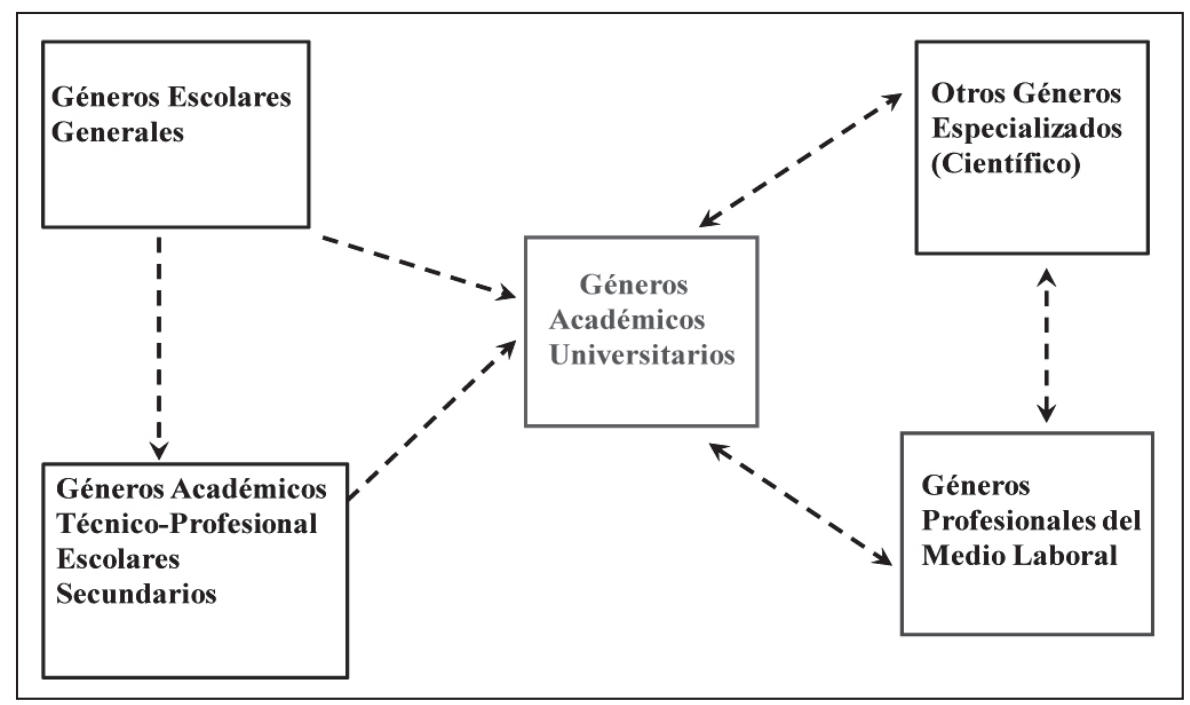

Figura 1. Contextos y géneros académicos y profesionales.

Así, conocer la diversidad de estos discursos constituye un área de particular interés desde el enfoque de la alfabetización disciplinar, tanto para los procesos de comprensión como de producción. Como se aprecia en esta figura, es muy posible que en la educación universitaria doctoral se encuentren géneros que provienen de otras esferas discursivas y que no todos sean originalmente producidos en el contexto académico propiamente tal. Justamente desde este análisis, Parodi (2008b), en base al estudio del Corpus PUCV-2006 en géneros de pregrado, propone que el discurso académico podría considerarse como un discurso mixto; lo que indicaría que en su constitución se combinan diversos géneros, construyéndose así una configuración heterogénea.

Ahora bien, respecto del concepto de género discursivo, existe hoy en día una amplia y rica discusión desde múltiples fuentes (Hyon, 1996; Parodi, 2008c; Bazerman, 2012; Bhatia, 2012; Martin, 2012; Miller, 2012; Swales, 2012, entre 
otros). En este sentido, las líneas contemporáneas de investigación revelan que no siempre existe consenso conceptual para abordar el estudio de los géneros y se detectan diferencias importantes entre las distintas escuelas de pensamiento, con énfasis muy divergentes en algunos casos; no obstante ello, es indiscutible el auge que concita este terreno de estudio, tal como se comprueba en publicaciones y congresos internacionales (Devitt, 2008; Martin y Rose, 2008; Bazerman, Bonini y Figueiredo, 2009; Bawarshi y Reiff, 2010).

En el contexto de esta investigación, adscribimos a la concepción multidimensional de género del discurso propuesta por Parodi (2008c), en la cual se destaca la interacción entre una dimensión lingüística, una social y una cognitiva. La siguiente figura intenta capturar esta dinámica.

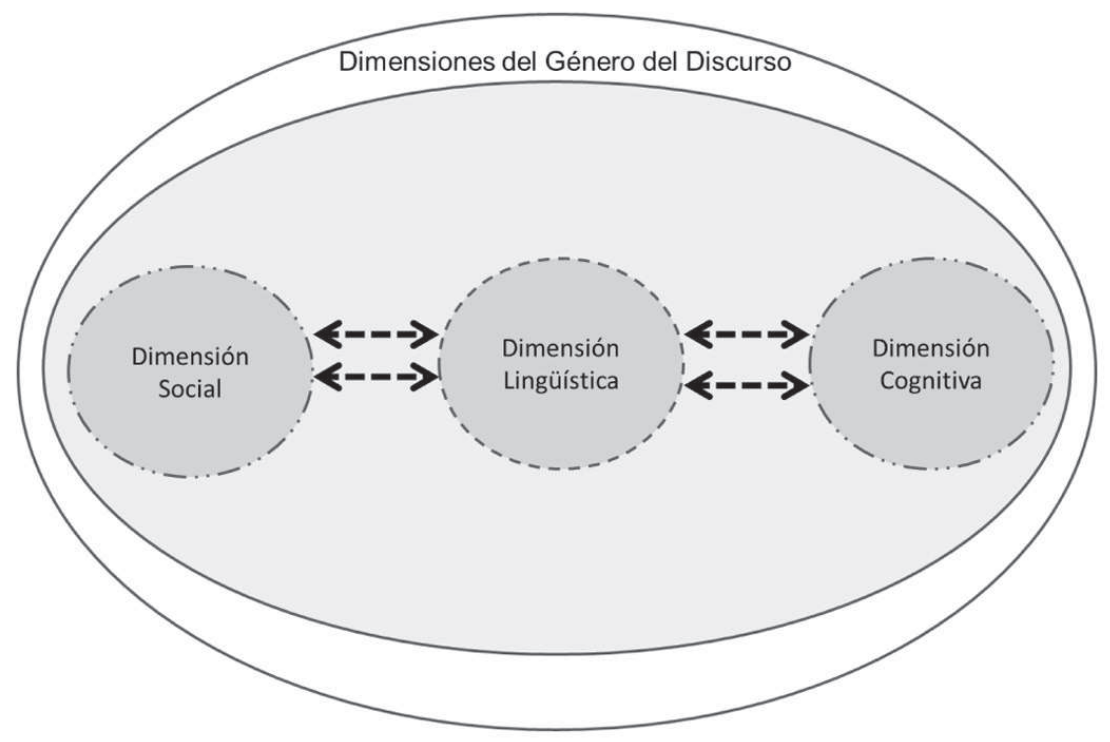

Figura 2. Diagrama de una concepción multidimensional triátida del género (Parodi, 2008c).

Desde esta concepción, sostenemos que los géneros son entidades complejas en que se actualizan discursivamente los propósitos comunicativos de escritores y hablantes en la interacción contextualmente situada, por medio de textos concretos que materializan los significados en elaboración. La dimensión lingüística ejerce, de este modo, un rol fundamental puesto que permite establecer la vinculación entre el plano contextual y el cognitivo. Ello implica que los significados en construcción se transmiten a través de enunciados particulares y los rasgos lingüísticos se disponen de modo singular al concretarse el género en instancias textuales. Así, estas entidades discursivas llamadas géneros, emergentes en situaciones sociales determinadas, logran cierta estabilidad, gracias a su naturaleza eminentemente cognitiva y su almacenamiento en la memoria de hablantes-oyentes y escritores- 
lectores. De este modo, estos modelos cognitivos de los formatos discursivos contextualmente construidos son activados y reelaborados cada vez que se busca llevar a cabo ciertos objetivos o propósitos comunicativos, enmarcados en situaciones sociales específicas.

En el caso del discurso académico, el estudio de los textos de un corpus, como instancias particulares de interacción social, permite identificar los rasgos lexicogramaticales que los caracterizan y, al estar aglutinados en torno a géneros, es posible construir un patrón de la cartografía lingüística de un género específico. Es, en este sentido, que sostenemos que los géneros cuentan con una dimensión lingüística, la cual es reconocible como un mapa geográfico o patrón organizacional que se constituye en la evidencia concreta de la manifestación de una entidad más abstracta. Esta cartografía, que puede incluir no sólo los patrones de rasgos lexicogramaticales (Parodi, 2005) sino también la organización retórico-funcional en términos -por ejemplo- de movidas (Parodi, 2008a; Ibáñez, 2008; Burdiles, 2012; Martínez, 2012), se constituye en un componente descriptivo fundamental si se busca aportar a la alfabetización disciplinar en el ámbito académico.

Ahora bien, en lo que respecta al discurso académico escrito universitario, estas entidades multidimensionales de interacción presentan una interesante diversidad, en donde se han identificado algunos que se crean específicamente en el entorno universitario y otros que se importan desde ámbitos más prototípicamente profesionales o científicos (Parodi, 2008b). También se han identificado géneros que despliegan patrones recursivos de tipo didáctico o divulgativo (por ejemplo, la Guía Didáctica y el Manual) y otros de prosa altamente especializada e informacionalmente densos (e.g., el Texto Disciplinar), así como otros que sólo se leen como fuente de acceso a información disciplinar y raramente se escriben en el contexto universitario (e.g., el Manual). Asimismo, las investigaciones en esta línea dan cuenta de géneros muy prototípicos que se escriben en entornos académicos universitarios de pre y postgrado, pero que también serán fundamentales en la vida del trabajo científico, como es el caso de la Reseña Crítica, el Artículo de Investigación y el Proyecto de Investigación (Parodi, 2008a; Jarpa, 2012).

\section{EL ESTUDIO: CONSTITUCIÓN DEL CORPUS Y PASOS METODOLÓGICOS}

\subsection{El Corpus PUCV-2010}

Tal como se ha descrito en Parodi (2010b), el Corpus PUCV-2010 se constituye a partir de los textos escritos que se entregan como lecturas obligatorias y complementarias a los alumnos de seis programas de doctorado en las áreas de las Ciencias Sociales y Humanidades (CS\&H) y de las Ciencias Básicas (CB). El corpus 
fue recolectado en doce programas de doctorado (dos por cada especialidad) en seis universidades chilenas, principalmente, a partir de análisis de los programas de las asignaturas obligatorias y optativas de cada doctorado, con la excepción de los materiales incluidos en la tesis doctoral. En la Tabla I se da cuenta de las seis disciplinas que constituyen la base para el Corpus PUCV-2010.

\begin{tabular}{|l|l|}
\hline \multicolumn{1}{|c|}{ Área } & \multicolumn{1}{c|}{ Disciplina } \\
\hline \multirow{2}{*}{$\begin{array}{l}\text { Ciencias Sociales y y } \\
\text { Humanidades }\end{array}$} & Historia \\
\cline { 2 - 2 } & Lingüística \\
\cline { 2 - 2 } Ciencias Básicas & Biotecnología \\
\cline { 2 - 2 } & Física \\
\cline { 2 - 2 } & Química \\
\hline
\end{tabular}

Tabla I. Disciplinas del Corpus PUCV-2010.

Estas disciplinas fueron seleccionadas con el objetivo de obtener una panorámica amplia de posibles variaciones en los documentos escritos provenientes de los respectivos programas de educación doctoral. Se espera así llegar a contar con un corpus diversificado que sustente estudios variacionistas en diversas líneas.

A continuación, en la Tabla II se registra el número de textos recolectados.

\begin{tabular}{|c|c|c|c|}
\hline \multicolumn{2}{|c|}{ Área y Disciplina } & $\begin{array}{c}\mathrm{N}^{\circ} \text { de Textos } \\
\text { por }\end{array}$ & $\begin{array}{c}\text { Totales por } \\
\text { Área }\end{array}$ \\
\hline \multirow{3}{*}{$\begin{array}{c}\text { Ciencias } \\
\text { Sociales y } \\
\text { Humanidades }\end{array}$} & Historia & 592 & \multirow{3}{*}{$2.603(82,7 \%)$} \\
\hline & Lingüística & 1.065 & \\
\hline & Literatura & 946 & \\
\hline \multirow{3}{*}{$\begin{array}{l}\text { Ciencias } \\
\text { Básicas }\end{array}$} & Biotecnología & 227 & \multirow{3}{*}{$544(17,3 \%)$} \\
\hline & Física & 181 & \\
\hline & Química & 136 & \\
\hline \multicolumn{2}{|c|}{ Total } & \multicolumn{2}{|c|}{$3.147(100 \%)$} \\
\hline
\end{tabular}

Tabla II. Constitución numérica del Corpus PUCV-2010.

Las cifras entregadas en la Tabla II dan cuenta de uno de los corpus académicos más extensos disponibles hoy en el mundo, si no el único. No tenemos registro 
de otro corpus, en cualquier lengua o lenguas, que se haya recopilado a partir de criterios rigurosos, principios ecológicos, con textos completos, temática y contextualmente circunscritos; esto es, textos que se leen y que constituyen el discurso académico a nivel de doctorado en seis disciplinas. Si a esto agregamos que los textos serán segmentados por género discursivo, contamos así con un corpus amplio y diversificado en disciplinas de CS\&H y CB.

El procedimiento de recolección de los textos del corpus ha sido relativamente similar al que hemos desarrollado en otras investigaciones precedentes y se resume en la tabla siguiente.

Tabla III. Formato empleado para la recolección y procesamiento del Corpus PUCV-2010.

\section{Pasos seguidos para recolectar el Corpus PUCV-2010}

Paso 1: Construcción de una base de datos con la información completa de los currículos de los programas de doctorado (incluyendo los programas de cada asignatura obligatoria y optativa).

Paso 2: Construcción de una base de datos con todas las referencias bibliográficas obligatorias y de consultas, incluidas en los programas de estudio.

Paso 3: Preparación de una encuesta para todos los profesores de cada uno de los seis programas, la cual incluye una solicitud de materiales complementarios no incluidos en los programas de asignaturas.

Paso 4: Recolección de material complementario a cada asignatura, que los profesores entregan a través de guías, archivos digitales y material fotocopiado.

Paso 5: Búsqueda en Internet con el fin de encontrar aquellos títulos disponibles en formato digital, minimizando así el tiempo de digitalización.

Paso 6: Recolección de los textos de las bibliotecas correspondientes y de las oficinas de los profesores.

Paso 7: Proceso de fotocopiado de cada texto con el fin de construir una base de datos en papel, para consultas posteriores.

Paso 8: Entrenamiento de un equipo de asistentes para escanear y compilar todos los textos.

\subsection{Metodología}

No cabe duda de que la identificación de géneros de discurso a partir del estudio de los textos concretos constituye una tarea compleja y que entraña una diversidad de pasos metodológicos, en torno a los cuales no se ha alcanzado un consenso definitivo. En parte, debido a que los marcos referenciales varían y los investigadores, provistos de estas herramientas conceptuales y de otras metodológicas, defienden 
unas y otras posturas. Persisten en este afán, por un lado, visiones más contextualistas y otras más cognitivistas; pero también, por otro lado, algunas más deductivistas y otras más radicalmente empiristas inductivistas (donde se puede aplicar la distinción de "basado en el corpus" y "guiado por el corpus").

Nuestra preocupación en estas cuestiones se remonta a la propuesta de Parodi y Gramajo (2003), orientada por los tipos de textos en un modelo multiniveles; posteriormente, desde la mirada multidimensional de los géneros del discurso en una concepción integral, Parodi, Venegas, Ibáñez y Gutiérrez (2008) avanzan en una propuesta de criterios y variables, conjugando enfoques deductivistas e inductivistas. Esto quiere decir que hemos, complementariamente, operacionalizado categorías conceptuales provenientes de la teoría, desde el conocimiento previo de los investigadores, y desde los datos empíricos emergentes de los textos desde una mirada más "guiada por el corpus" (Tognini-Bonelli, 2001; Biber, Connor y Upton, 2007). De modo que la complementariedad de enfoques es un asunto central para alcanzar, en nuestro caso, una mejor y más acotada distinción entre los géneros del corpus.

Entonces, para el presente estudio seguimos la propuesta elaborada por Parodi et al. (2008), la cual se cimenta en cinco criterios fundamentales: a) macropropósito comunicativo, b) modo de organización del discurso, c) relación entre los participantes, d) contexto ideal de circulación y e) modalidad. Estos criterios, a su vez, se especifican en veintiséis variables.

Tal como decíamos más arriba, los cinco criterios centrales se especificaron en un conjunto de variables. Estas variables se analizan en los textos bajo el principio de "predominancia" de una sobre otra. Esto quiere decir que, desde nuestra concepción multidimensional, estamos conscientes de que los textos y los géneros no son unidades totalmente homogéneas ni estables. En ellos, por ejemplo, coexisten diversos macropropósitos comunicativos y a lo largo de sus pasajes se intercalan múltiples modos de organización discursiva.

\section{RESULTADOS Y DISCUSIÓN}

\subsection{Identificación de géneros y cifras generales}

En la primera parte de la presentación de los resultados entregamos el listado de los 30 géneros que emergieron del análisis realizado por el equipo de investigación del Proyecto FONDECYT 1090030, siguiendo la matriz de Parodi et al. (2008). Este análisis se llevó a cabo sobre la totalidad del Corpus PUCV-2010, esto es, estudiando los 3.147 textos recolectados en las seis disciplinas. La Tabla IV muestra este resultado. 
Tabla IV. Géneros identificados en el Corpus PUCV-2010.

\begin{tabular}{|l|l|l|l|l|l|}
\hline $\mathbf{1}$ & Acta & ACT & $\mathbf{1 6}$ & Índice de Resúmenes Gráficos & IRG \\
\hline $\mathbf{2}$ & Antología & ANT & $\mathbf{1 7}$ & Informe & INF \\
\hline $\mathbf{3}$ & Artículo de Investigación & AI & $\mathbf{1 8}$ & Manifiesto & MTO \\
\hline $\mathbf{4}$ & Artículo de Opinión & AOP & $\mathbf{1 9}$ & Manual & MA \\
\hline $\mathbf{5}$ & Atlas & ATL & $\mathbf{2 0}$ & Nota Científica & NOT \\
\hline $\mathbf{6}$ & Biografía & BIO & $\mathbf{2 8}$ & Nota Técnica & TN \\
\hline 7 & Cuentos & CUE & $\mathbf{2 1}$ & Novela & NOV \\
\hline $\mathbf{8}$ & Diccionario & DC & $\mathbf{2 3}$ & Obra Dramática & ODR \\
\hline $\mathbf{9}$ & Discurso de Agradecimiento & DAG & $\mathbf{2 2}$ & Obras Completas & OCO \\
\hline $\mathbf{1 0}$ & Editorial & EDI & $\mathbf{2 4}$ & Poema & POE \\
\hline $\mathbf{1 1}$ & Epistolario & EPI & $\mathbf{2 5}$ & Referencia Bibliográfica & REB \\
\hline $\mathbf{1 2}$ & Errata & ERR & $\mathbf{2 6}$ & Reportaje Científico & REC \\
\hline 13 & Evangelio & EVA & $\mathbf{2 7}$ & Reseña & RES \\
\hline $\mathbf{1 4}$ & Gabinete & GAB & $\mathbf{2 9}$ & Tesis & TES \\
\hline $\mathbf{1 5}$ & Guía Didáctica & GD & $\mathbf{3 0}$ & Texto Disciplinar & TD \\
\hline
\end{tabular}

Este primer hallazgo empírico muestra una amplia gama de géneros, los cuales pasaron una serie de fases de análisis para llegar a esta clasificación final. En general, la tendencia fue a la reducción del número de géneros, buscando evitar la proliferación innecesaria y la subespecificación de géneros a partir del discurso en ciertas disciplinas. Tal como en estudios anteriores de nuestro equipo en esta línea de investigación (Parodi, 2008a), para la determinación de los nombres de los géneros se buscó etiquetas relativamente simples, intentando -la mayoría de las veces- que ellas correspondieran a los usos más regulares. Siempre que se dio el caso, mantuvimos los nombres determinados en investigaciones anteriores ( $\mathrm{Pa}$ rodi y Gramajo, 2003; Parodi, 2004, 2008a, 2009); en particular, de aquellos en que existe amplio consenso, e.g., Artículo de Investigación (AI), Manual (MA), y de otros que fueron propuestos y definidos por Parodi et al. (2008), como es el Texto Disciplinar (TD). En cuanto a nuevos géneros, buscamos nombres que la comunidad discursiva emplea o los que se identifican en los textos mismos (por ejemplo, Índice de Resúmenes Gráficos y Nota Técnica).

Resulta interesante destacar que el número de 30 géneros es mayor que el identificado en investigaciones anteriores basadas en los corpus PUCV-2003 y PUCV2006 (Parodi y Gramajo, 2003; Parodi, 2004, 2008a, 2009). En parte, ello puede explicarse debido a que es primera vez que investigamos en el discurso académico de seis disciplinas y, como es claro, en el nivel de doctorado. Estas dos variables en conjunto permiten, posiblemente, explicar el mayor número de géneros detectados en esta investigación. 
Ahora bien, una vez identificados los géneros a partir del análisis de los 3.147 textos del Corpus PUCV-2010, procedimos a su cuantificación, pues nos interesaba conocer la distribución con que ellos ocurren en el corpus. A continuación, en el Gráfico 1, se entrega un primer cálculo general, expresado en porcentajes.

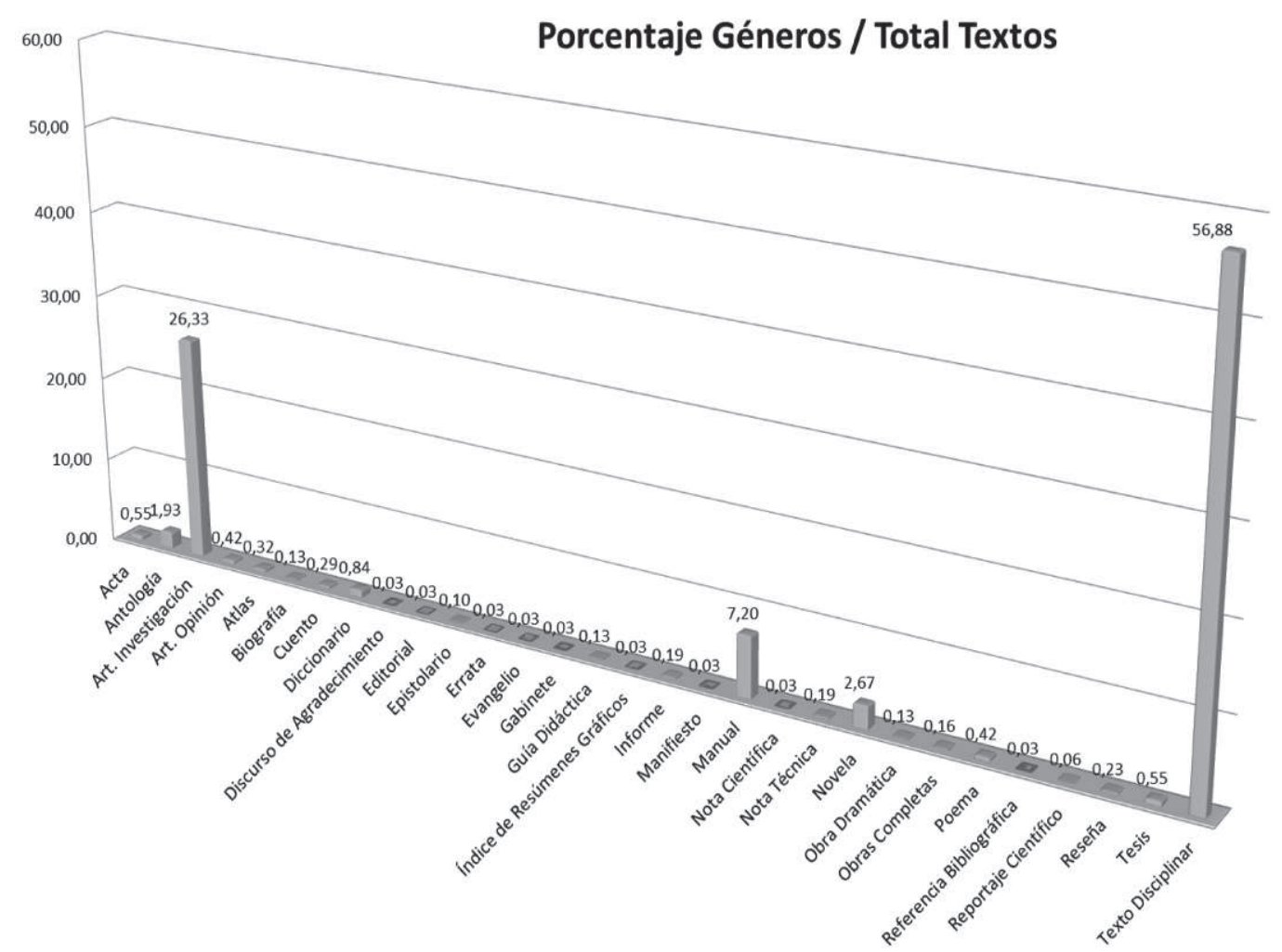

Gráfico 1. Cuantificación general del total de géneros identificados.

Las cifras mostradas a través de este gráfico son muy reveladoras y aportan información fundamental acerca del corpus académico y los géneros identificados. En primer lugar, cabe destacar que, a pesar de la importante e interesante heterogeneidad de géneros discursivos que constituyen este corpus de discurso académico en estas seis disciplinas, se hace evidente que no son tan variados los mecanismos o formatos discursivos más recurrentes en que se comunica el conocimiento especializado a nivel de doctorado. Es muy significativo notar que son únicamente dos los géneros principales: TD con la más alta ocurrencia de 57\%, y AI con 26\%; en tercer lugar los sigue, a distancia importante, el MA con un 7\%.

Por su parte, los géneros Novela y Antología registran las únicas otras dos cifras con relativa importancia en cuarto y quinto lugar, esto es, con un 3 y $2 \%$ respectivamente. Resulta interesante descubrir la importante presencia de estos dos géneros claramente de naturaleza literaria como parte del discurso académico a nivel de doctorado; más adelante, en el estudio pormenorizado de los géneros en cada discurso disciplinar, comentaremos acerca de su posible función en el corpus. 
Los otros veinticinco géneros restantes, identificados en el Corpus PUCV-2010, alcanzan porcentajes de ocurrencia que no superan el $1 \%$ y, en muchos casos, se acercan mucho a cero; hecho que quiere decir que algunos o muchos de estos géneros sólo cuentan con unos pocos ejemplares en este corpus de 3.147 textos.

Ahora bien, basados en estas cifras, resulta indudable que el discurso académico en estas disciplinas no se constituye -de modo fundamental- por una amplia ni variada gama de recursos genéricos, sino, muy por el contrario, los textos del corpus se aglutinan en torno a algunos formatos discursivos, al parecer muy idiosincráticos y fácilmente reconocibles: TD, AI y MA. En otras palabras, los hallazgos aquí reportados muestran que el conocimiento especializado en estas seis disciplinas se comunica y se posibilita su transmisión y construcción, fundamentalmente, por medio de géneros claramente focalizados en tres tipos de interacciones discursivas:

a) elaboración de teorías, reflexiones conceptuales, discusiones teórico-metodológicas, y comunicación de hallazgos empíricos, como es el Texto Disciplinar;

b) comunicación de resultados investigativos y hallazgos empíricos (por medio del Artículo de Investigación) y

c) comunicación y enseñanza de conceptos teóricos y aplicados, modelización de procedimientos, presentación de problemas y resolución paso a paso, como sucede en el Manual.

A continuación proporcionamos una definición de estos tres géneros, basados en la matriz de análisis elaborada por Parodi et al. (2008). Ellos permiten ilustrar, por un lado, el modo en que se construyen estas definiciones según los criterios y variables de la matriz en cuestión y, por otro, muestran los rasgos de cada uno de estos tres géneros:

Texto Disciplinar (TD): Género discursivo cuyo macropropósito comunicativo es persuadir respecto del tratamiento de uno o varios temas de una disciplina particular. Su contexto de circulación es el ámbito científico y la relación de los participantes es entre escritor experto y lector experto. Preferentemente, se hace uso de un modo de organización discursiva argumentativo. También se emplean recursos multimodales.

Artículo de Investigación (AI): Género discursivo cuyo macropropósito comunicativo es persuadir respecto de un determinado punto de vista, ya sea por medio de una revisión teórica o respecto de los resultados obtenidos en un estudio empírico. Idealmente, su contexto de circulación es el ámbito científico y la relación entre los participantes es entre escritor experto y lector experto. Preferentemente, se hace uso de un modo de organización discursiva, predominantemente, argumentativo y con apoyo de recursos multimodales. 
Manual (MA): Género discursivo cuyo macropropósito comunicativo es instruir acerca de conceptos y/o procedimientos en una temática especializada. Su contexto de circulación ideal es el ámbito pedagógico y la relación entre los participantes es entre escritor experto y lector semilego o lego. Preferentemente, se hace uso de un modo de organización discursiva descriptivo y con recursos multimodales.

Ahora bien, con el fin de estudiar con mayor detalle las cifras generales del Gráfico 1, procedemos a graficarlas ahora por disciplina.

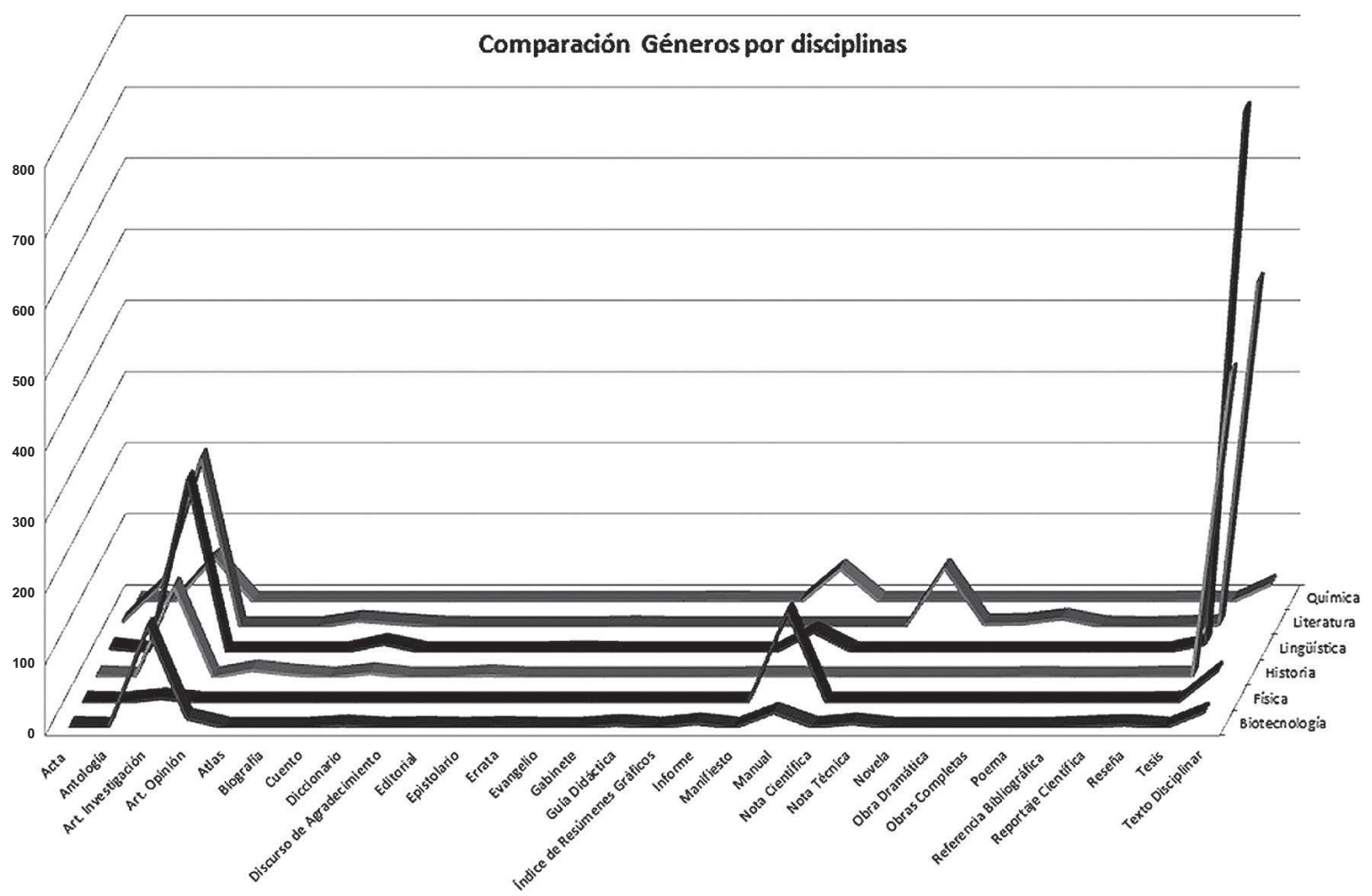

Gráfico 2. Ocurrencia de géneros por disciplina (cifras brutas).

Si las cifras del Gráfico 1 resultaron muy reveladoras, las que se aprecian en este segundo gráfico se consideran altamente informativas y reconfirman nuestras apreciaciones en cuanto a la amplia variación genérica, pero escasa diversidad de los medios discursivos fundamentales y más prototípicos del discurso académico disciplinar. Así, por una parte, destacamos que el TD es un género altamente importante en el discurso académico de las disciplinas en indagación, tal como se comprueba en las cifras brutas mostradas en este segundo gráfico (más de 700 ejemplares en Lingüística, cerca de 600 en Literatura y por sobre los 400 en Historia). Queda en evidencia que el TD se establece como un género fundamental para la transmisión de información especializada y resulta el soporte imprescin- 
dible que posibilita la construcción de conocimientos disciplinares en estas tres disciplinas de CS\&H a nivel de doctorado. Complementariamente, el rol singular del TD en CS\&H es coincidente con los hallazgos reportados por Parodi (2008d) en un corpus de textos académicos universitarios de pregrado en las disciplinas de Psicología y Trabajo Social.

Por otra parte, las cifras del Gráfico 2 también revelan que el AI y el MA son géneros que ocurren a través del discurso académico de varias de las disciplinas abordadas en este estudio. Aunque las cifras brutas muestran su menor ocurrencia en comparación con el TD, el número de ejemplares no deja de ser significativo: en algunos casos por sobre $200 \mathrm{y}$, en otros, cerca de 100.

\subsection{Los géneros en el discurso de cada una de las seis disciplinas}

En lo que sigue damos cuenta de los porcentajes de ocurrencia de los géneros identificados en el discurso académico de cada una de las seis disciplinas. Veamos, en primer lugar, los resultados para las tres disciplinas de $\mathrm{CB}$; en particular, de Química.

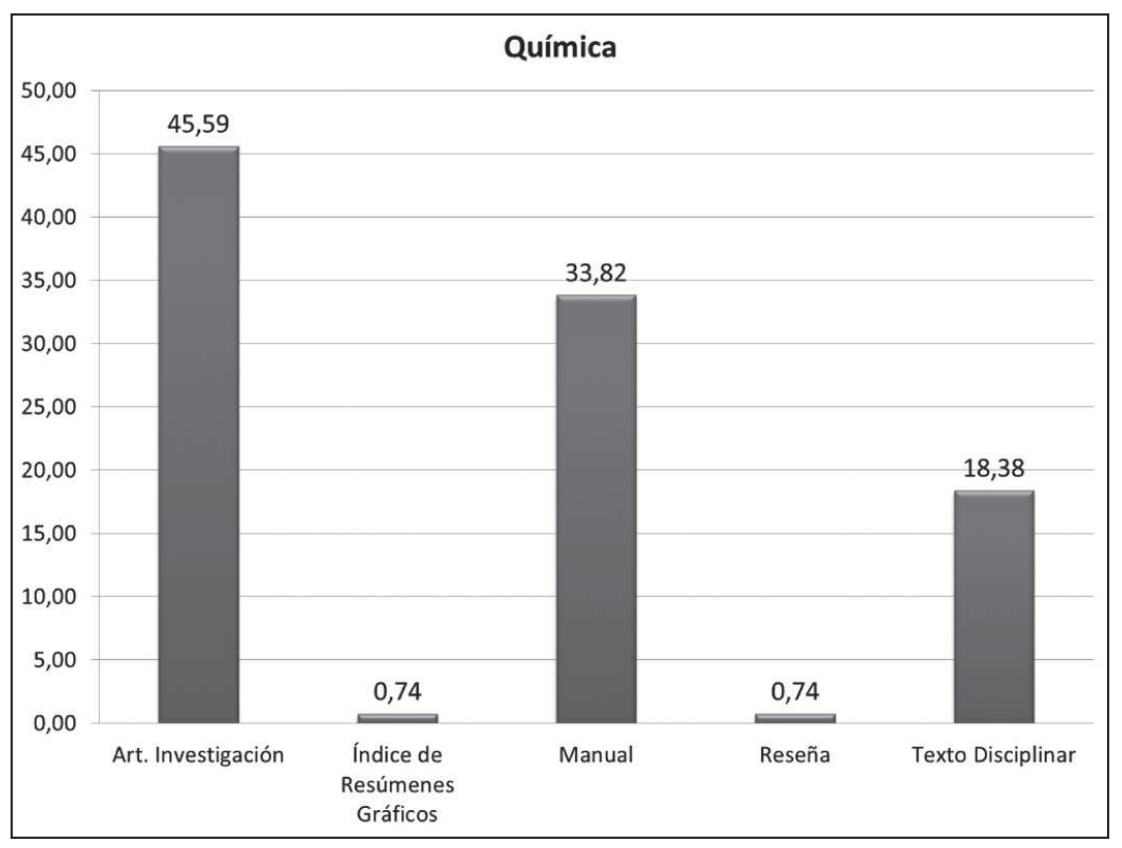

Gráfico 3. Géneros en la disciplina de Química.

Tal como se muestra en este gráfico, son cinco los géneros reconocidos a partir de los 136 textos recolectados en la disciplina doctoral de Química. Por orden de mayor a menor ocurrencia, ellos son: AI, MA, TD, RES e IRG. Se hace evidente que el AI, el MA y el TD se constituyen en los más prototípicos y fundamentales y que los otros dos desempeñan un rol muy menor $(0,7 \%)$ y claramente satelital o 
marginal. Resulta interesante que en la transmisión de conocimientos se despliegan recursos informativos altamente especializados (AI y TD), pero también se encuentra otro más didáctico e instruccional (MA), en el que se emplean estrategias divulgativas y reformulativas. En este último género, Parodi (2008d) ha descrito su función fundamental en disciplinas como Química Industrial e Ingeniería en Construcción para la enseñanza de conceptos y procedimientos, por medio de la ejemplificación y resolución de problemas en formatos muy ilustrativos. Los datos ahora aportados para el nivel doctoral indican la combinación de géneros y la tendencia hacia la mayor especialización.

En el Gráfico 4 se entregan los resultados numéricos por género para la disciplina de Física.

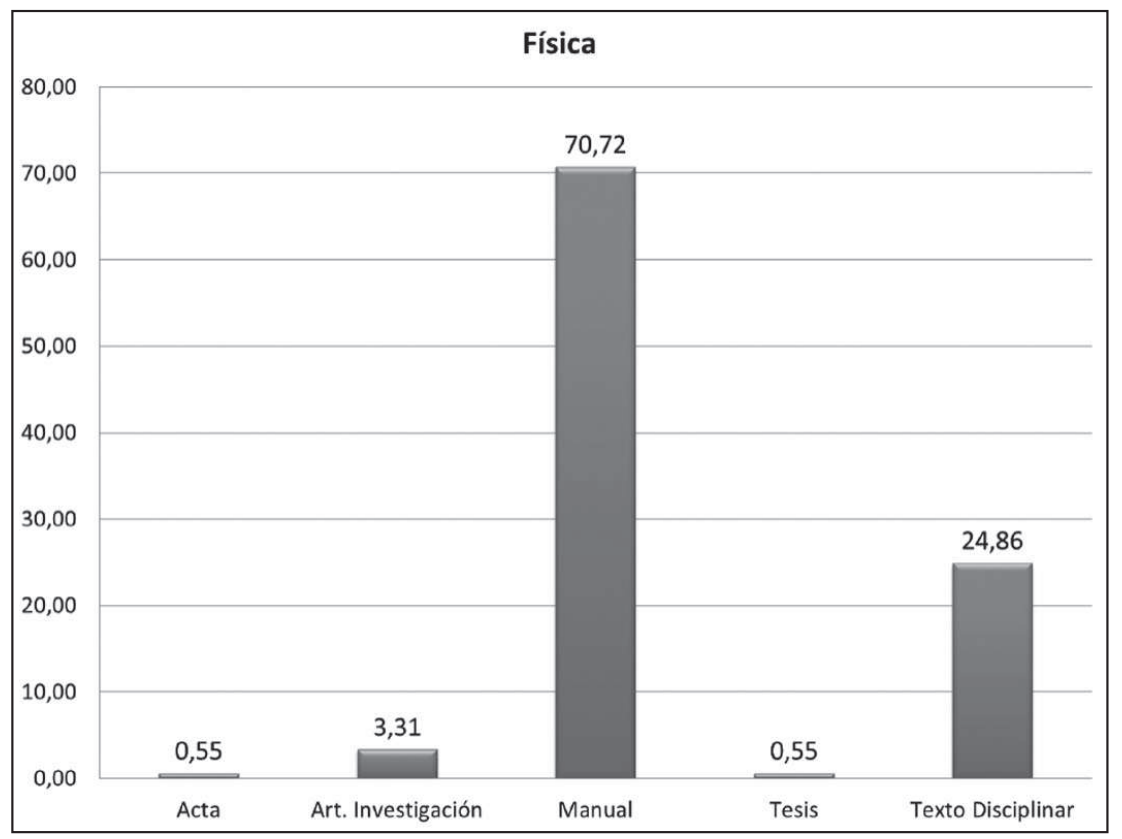

Gráfico 4. Géneros en la disciplina de Física.

Las cifras de este gráfico muestran que -al igual que en Química- son cinco los géneros identificados en base a los 181 textos de este corpus, pero los géneros no son todos los mismos ni su ocurrencia porcentual es similar. Los tres más importantes en este caso son el MA (71\%), el TD (25\%) y el AI (3\%). Los dos restantes, Acta y Tesis, alcanzan ambos un 0,5\%. En el discurso de esta disciplina también se alternan géneros en que se entregan conocimientos altamente especializados. La relación entre participantes puede ser catalogada de experto a experto, como ocurre en el AI y el TD; no obstante ello, es el género Manual el que se revela como la herramienta discursiva por excelencia en el acceso a los contenidos disciplinares, a través del cual se despliegan estrategias didácticas de definición de conceptos especializados, ejemplificación, clasificación y presentación de problemas con sus 
respectivas resoluciones y modelizaciones. En este género, la relación entre los participantes en el discurso es de experto a semilego. Todo esto quiere decir que el $71 \%$ de los textos del corpus de Física se identifican como pertenecientes a un género especializado, pero que andamia progresivamente el acceso a la información y posibilita así la construcción del conocimiento disciplinar; todo ello de modo muy pedagógico y con el propósito de instruir a audiencias en formación.

Ahora bien, el hecho de que el género Manual sea el preponderante en esta disciplina no implica de modo alguno que su contenido disciplinar muestre menor rigor científico ni alcance grados importantes de especialización y profundidad. Lo que revela este hallazgo es un modo de presentar el conocimiento experto de forma especial, a través de mecanismos discursivos que indican una preocupación singular por el lector y el aprendiente.

A continuación, en el Gráfico 5 se presentan los catorce géneros que se registran en el discurso de la disciplina de Biotecnología.

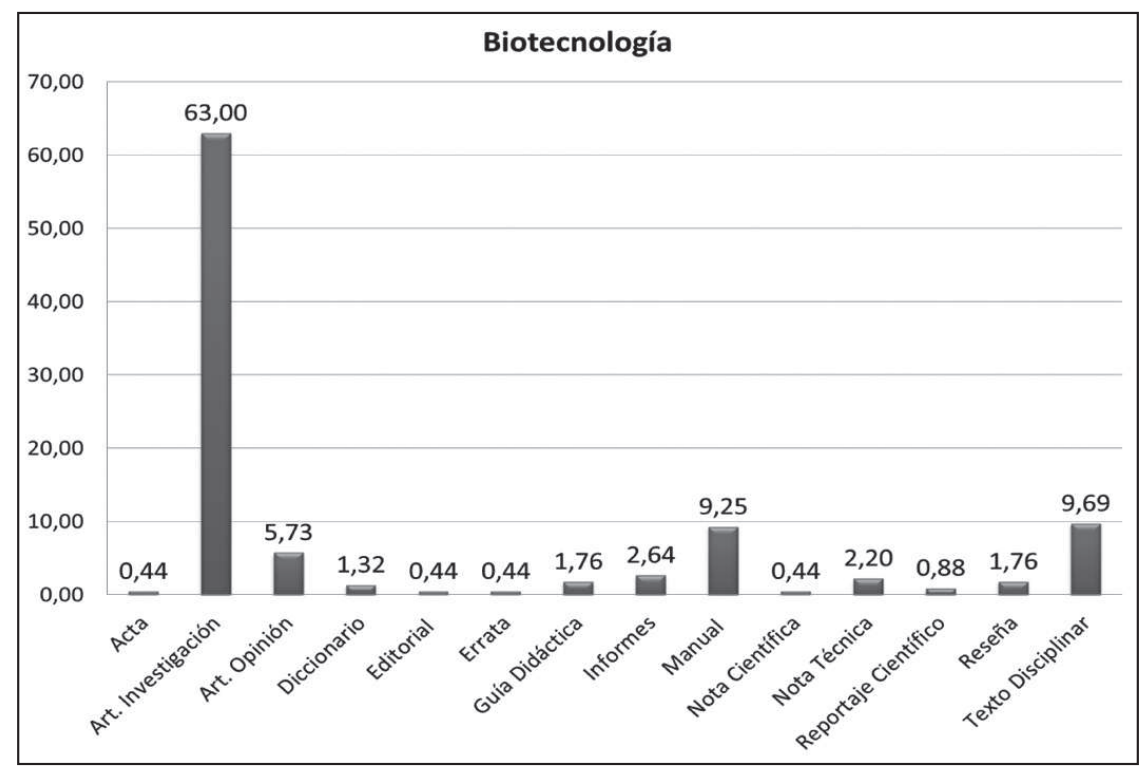

Gráfico 5. Géneros en la disciplina de Biotecnología.

Tal como se muestra en este gráfico, el número de géneros en el discurso de Biotecnología es considerablemente más amplio que en las otras dos disciplinas de las Ciencias Básicas. A pesar de la mayor diversidad de géneros identificados en el corpus de 227 textos de Física, en comparación con Química y Física, son los mismos tres géneros detectados como de mayor ocurrencia en estas otras dos disciplinas los que en Física también muestran los más altos porcentajes: AI (63\%), TD (10\%) y MA (9\%). Los otros once géneros aparecen con escasa o muy baja presencia. 
Ahora bien, a diferencia del discurso de Física, estos tres géneros en Biotecnología se ordenan jerárquicamente de modo distinto a lo que se exponía más arriba en cuanto a recursos discursivos didácticos. En este caso, el AI es el género que se muestra más relevante y que ofrecería el acceso a la información y andamiaría la construcción del conocimiento especializado. Como se sabe, en este género se dispone información entre expertos, se comunica el conocimiento de vanguardia y se exponen hallazgos científicos de naturaleza teórica y empírica. El macropropósito comunicativo es persuadir por medio de diversas estrategias argumentativas. Junto al AI, se detecta un segundo género especializado en el que también se da una relación experta entre escritor y lector, este es el TD.

Por su parte, el MA también tiene un lugar relevante en el discurso de la Biotecnología, aunque en tercer lugar de ocurrencia porcentual (9\%). Su importante ocurrencia muestra que se conjugan diversos tipos de géneros en el grado de didacticidad, atendiendo también así a la formación de audiencias -en este casosemilegas.

La importante heterogeneidad de géneros en el discurso de esta disciplina abre un interesante escenario que permite visualizar géneros como el Artículo de Opinión, la Nota Técnica, el Informe, la Reseña, la Guía Didáctica y el Diccionario. Todos ellos con ocurrencias por sobre el 1\%. Esta amplia diversidad de géneros en Biotecnología (14 en total) hará que esta disciplina se constituya -tal como se verá más adelante- en la segunda mayoría en heterogeneidad en el corpus PUCV2010 .

Los siguientes gráficos dan cuenta de la cuantificación de los géneros identificados en las tres disciplinas en estudio de CS\&H. En primer lugar, revisaremos los géneros que se registran en el discurso de Lingüística.

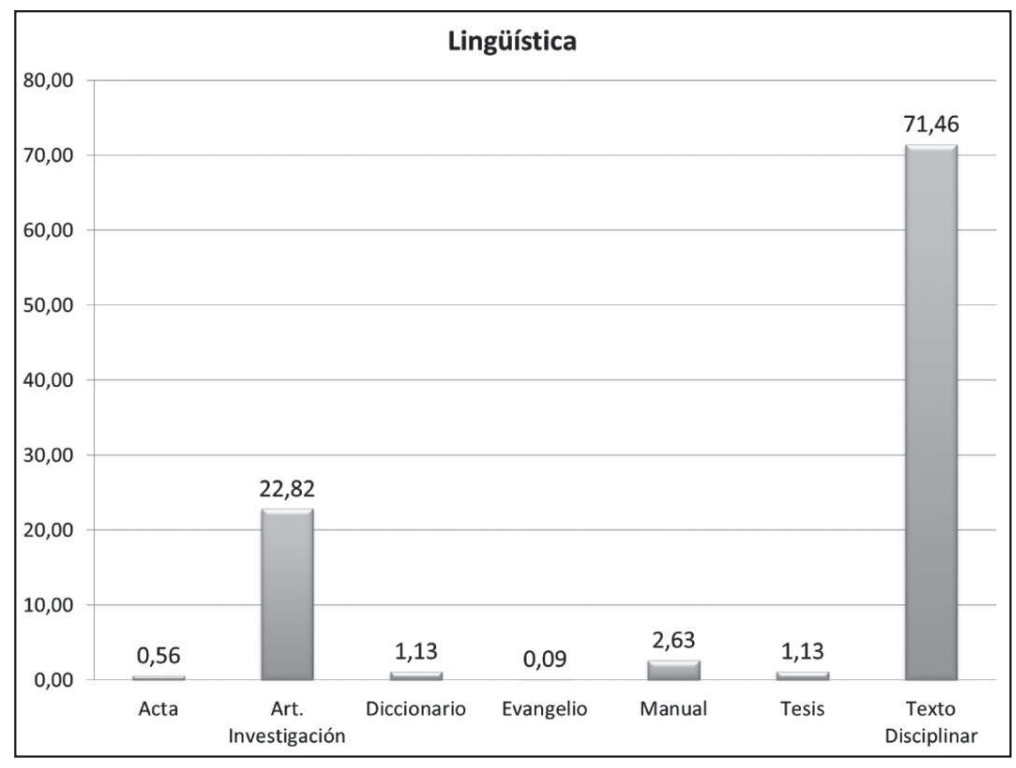

Gráfico 6. Géneros en la disciplina de Lingüística. 
El análisis de los 1.065 textos del corpus del discurso de la formación doctoral en Lingüística logró identificar siete géneros. De estos siete géneros, solo dos presentan una ocurrencia importante: TD (71\%) y AI (23\%). Ambos géneros son prototípicos de la transmisión del conocimiento altamente especializado. La escasa ocurrencia del género MA (3\%), a diferencia de lo que sucede en el discurso de las tres disciplinas indagadas en $\mathrm{CB}$, muestra que éste no representa un mecanismo discursivo prototípico en esta área del conocimiento. Investigaciones anteriores a partir del Corpus PUCV-2006 ya habían puesto de manifiesto esta diferencia entre el discurso académico en carreras de pregrado de CS\&H y el discurso académico de pregrado de las Ciencias Básicas y de la Ingeniería (Parodi, 2008d; Ibáñez, 2008). Ahora, a partir de los datos empíricos hasta aquí presentados, podemos mostrar que esta diferencia también se produce en el discurso de formación universitaria doctoral. El TD es relevante en CS\&H, lo mismo que el MA es relevante en CB.

Del mismo modo, aunque no con la misma alta ocurrencia, el AI se constituye en el segundo género fundamental en la formación doctoral en la disciplina de Lingüística. En este sentido, queda claro que en esta disciplina los conocimientos se transmiten primordialmente por medio de libros, seguidos en ocurrencia porcentual por artículos publicados en revistas científicas, editadas tanto en formato papel como digital.

Cabe señalar que la identificación del Evangelio como un género de este corpus resultó un hallazgo interesante. Su ocurrencia, sin duda, obedece a un texto empleado en algún tipo de análisis textual, más que a un texto de estudio disciplinar.

En esta misma línea de los datos anteriores, las cifras del Gráfico 7 dan cuenta de la importante diversidad de géneros que se identifican en el discurso doctoral de la formación en Historia. Trece géneros se registran, en donde el TD ostenta el $73 \%$ de ocurrencia.

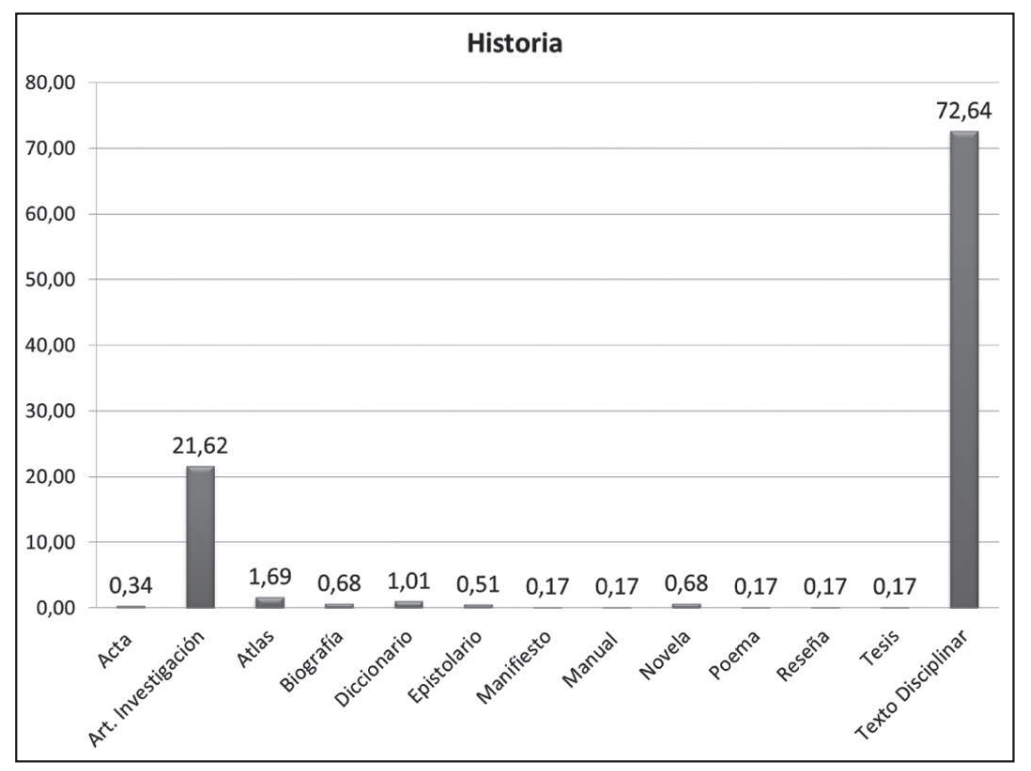

Gráfico 7. Géneros en la disciplina de Historia. 
Tal como se aprecia en los datos de este gráfico, de los trece géneros aquí presentados en base a los 502 textos recolectados, sólo dos registran una ocurrencia importante: TD (73\%) y AI (22\%). De los otros once géneros, sólo dos sobrepasan escasamente el 1\% de ocurrencia: Atlas y Diccionario.

Aunque se detecta una importante variación genérica en el discurso académico doctoral de Historia, su composición en términos de altas ocurrencias se acerca a la descubierta en el discurso académico de Lingüística; claro que este último con una muy menor heterogeneidad. Así, el discurso académico especializado en Lingüística e Historia revela mecanismos discursivos similares en el acceso a información disciplinar; no obstante ello, a pesar de compartir géneros similares, se hace fundamental un estudio pormenorizado y profundo de los textos que componen estos géneros a través de las disciplinas. La sola pertenencia a un género no constituye garantía definitiva de que los textos se constituyan de modo idéntico ni de que las organizaciones retórico-funcionales cumplan los mismos roles. Al respecto, Parodi (2008d) e Ibáñez (2008) demostraron, por medio del estudio de las movidas retóricas, que los géneros MA y TD varían en su constitución a través del discurso de ciertas disciplinas; en particular, de algunas de CS\&H y de CB.

No obstante las posibles variaciones en términos comparativos entre géneros en diversas disciplinas, es muy significativo identificar que -en estas dos disciplinas- el libro impreso se posiciona como el mecanismo discursivo de más alta ocurrencia y muy por sobre el siguiente género (AI), el cual se difunde, a través de textos de extensión relativamente breve, en revistas especializadas tanto en papel como en formato electrónico.

Especial mención merecen los géneros Epistolario, Novela y Poema dentro del corpus de Historia. La presencia de estos géneros apunta hacia dos posibles tipos de discurso dentro del corpus: uno que analiza y se basa en determinados textos y otro que se constituye en el objeto de análisis. Esta doble articulación de géneros muestra la naturaleza eminentemente discursiva de la disciplina. Esto quiere decir que se detectan, por un lado, géneros objeto de estudio e investigación y, por otro, géneros que presentan el análisis y la reflexión sobre la base de los primeros. En otras palabras, existen géneros que son fuente de información o materia de estudio y otros que -en parte- se sustentan en lo que aportan los géneros fuente.

En último lugar, se presenta el análisis de los 946 textos del corpus del discurso de Literatura, a partir del cual se detectó un total de quince géneros. 


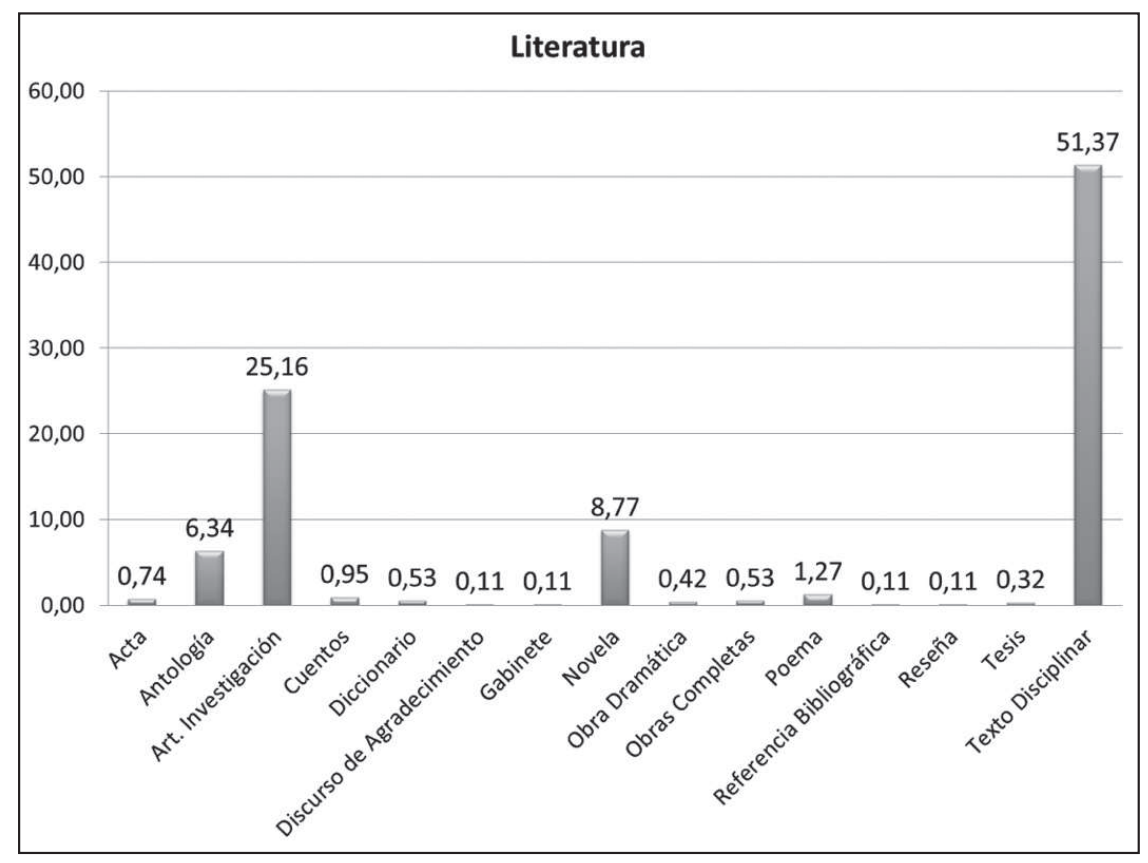

Gráfico 8. Géneros en la disciplina de Literatura.

El estudio de este corpus disciplinar reveló que el discurso académico de Literatura es el de mayor heterogeneidad genérica. En términos de ocurrencias, también se comprueba que son dos los géneros preferidos, al igual que en Lingüística e Historia: Texto Disciplinar (51\%) y Artículo de Investigación (25\%). Con este último hallazgo queda de manifiesto que el discurso de estas tres disciplinas de CS\&H comparte una singularidad en torno a la alta especialización y a géneros en donde la relación entre los participantes escritores y lectores es de experto a experto. Como es fácil comprobar a partir de las cifras entregadas, escasa o nula es la inclusión de géneros con un marcado acento divulgativo o con despliegue de estrategias didácticas como parte del discurso académico de doctorado. El Manual no se detecta en Literatura y su ocurrencia es bajísima o casi nula en Historia y Lingüística.

Un hallazgo que llama la atención en los géneros identificados en el corpus de Literatura es la presencia de géneros que podríamos llamar literarios propiamente tales, e.g. Antología, Novela, Cuento, entre otros; los cuales -ciertamente- se perciben diferentes al Texto Disciplinar y el Artículo de Investigación. Sobre esta base $\mathrm{y}$ con el fin de indagar en estas posibles distinciones, aglutinamos los textos que pertenecen a los géneros literarios y a los no literarios. En el Gráfico 9 se entrega el porcentaje de su ocurrencia. 


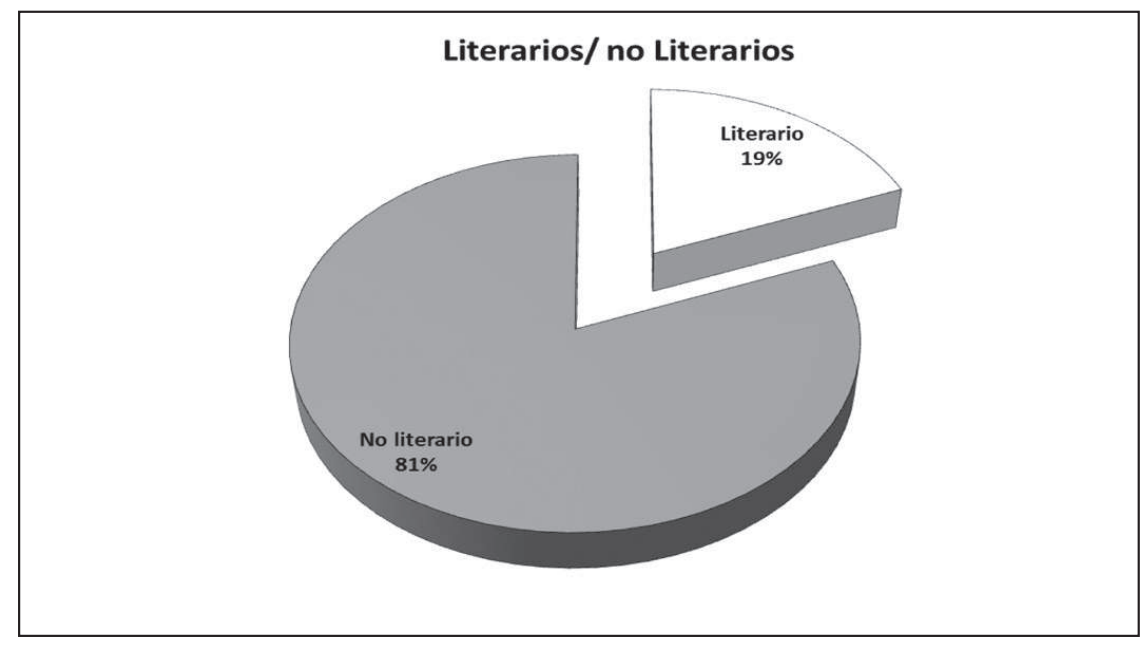

Gráfico 9. Distinción entre géneros literarios y no literarios (porcentaje global).

Como es fácil observar en este gráfico, un $81 \%$ de los géneros es no literario, esto es, presentan información en que se describe y reflexiona acerca de teorías, modelos o propuestas conceptuales y se analiza textos que pertenecen a los llamados géneros literarios. Por su parte, el 19\% de los géneros restante está constituido por textos que se inscriben en los considerados literarios. Esto quiere decir que el $19 \%$ de estos géneros literarios del discurso académico de Literatura se constituyen en la materia de estudio e investigación en el marco de estos programas de doctorado, lo que se plasma en los otros géneros no literarios.

A la luz de estos hallazgos, la distinción aquí mostrada se hace muy relevante y permite ilustrar una característica singular del discurso académico especializado de la Literatura en que se aprecia una separación fundamental entre dos tipos de géneros. Esta constitución genérica del corpus de Literatura revela un rasgo particular que sólo se detecta de modo menor en este corpus de Historia. Así, existen géneros académicos que se crean para comunicar la reflexión y el análisis (Texto Disciplinar, Artículo de Investigación, Tesis, Reseña, entre otros) a partir de otros géneros (Novela, Poema, Cuento, Obra Completa, entre otros), los cuales son la materia prima que dan origen y permiten la existencia de los otros.

Como una forma de profundizar en estos datos, calculamos otra vez el porcentaje de ocurrencia de los géneros identificados, pero ahora como parte de los macrogéneros literario y no literario. 


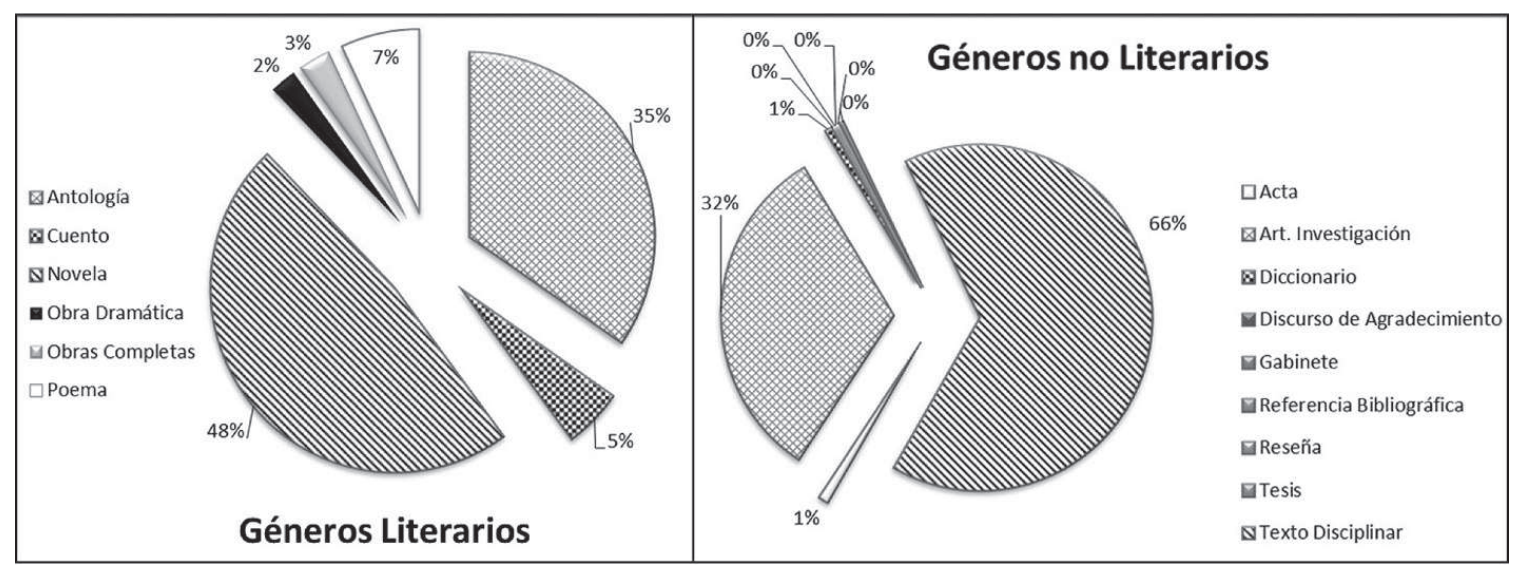

Gráfico 10. Comparación entre géneros literarios y no literarios.

Como se muestra en el Gráfico 10, la Novela (48\%) y la Antología (35\%) son los géneros más ocurrentes en su naturaleza literaria, seguidos por el Poema y el Cuento. Ello revela que los textos de predominancia narrativa son los preferidos como materia de estudio y análisis a nivel de doctorado, aunque la poética también se revela como un género importante en este nivel de estudios doctorales.

Por su parte, la reflexión e indagación literaria se lleva a cabo fundamentalmente por medio del Texto Disciplinar (66\%) y el Artículo de Investigación (32\%). Ambos géneros revelan alta especialización y escasas técnicas de divulgación. Otros géneros no literarios registrados en este corpus muestran una muy menor ocurrencia, tan escasa que sólo uno de ellos alcanza el 1\% (Acta) y que también comparte rasgos de alta especialización con el TD y el MA.

Ahora bien, a modo de discusión general y con el fin de poder apreciar cómo el total de los géneros identificados en el Corpus PUCV-2010 se comparten o no entre el discurso de las seis disciplinas en estudio, es decir, para identificar semejanzas y diferencias en su pertenencia a un dominio especializado y otro, se procede a diagramarlos por medio de intersecciones ilustrativas. 


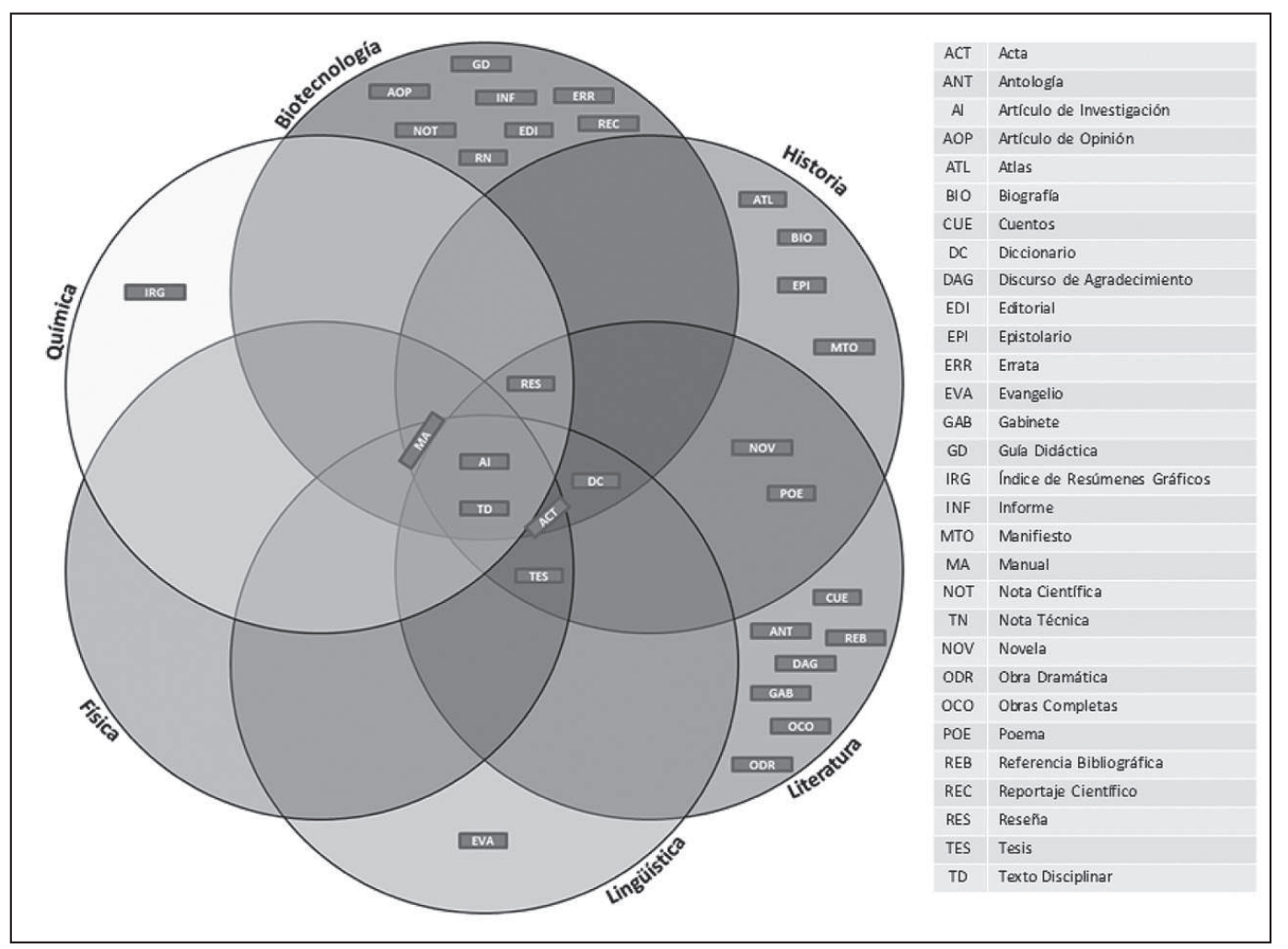

Figura 3. Intersección de géneros a través de las seis disciplinas.

El diagrama de Venn permite visualizar con claridad los 30 géneros del discurso identificados en su distribución a través del discurso académico de las seis disciplinas, destacando aquellos que se comparten entre dos o más disciplinas y aquellos que son exclusivos de un dominio especializado y otro. También se aprecia aquella disciplina que no cuenta con un género único (Biotecnología).

Un primer dato relevante lo constituye la identificación del TD y el AI como los dos géneros que ocurren en el discurso de las seis disciplinas del corpus. Este hallazgo muestra que el libro especializado en formato papel y el artículo publicado en revistas disciplinares, tanto en formato papel como electrónico, se posicionan como dos géneros que tienen formatos diversos y medios de circulación también variados en la comunicación del conocimiento disciplinar. Ello pone de manifiesto que en estas seis disciplinas, a pesar de la mayor o menor diversidad genérica, el conocimiento especializado se comunica primordialmente a través de recursos discursivos que operan transversalmente. Esto indica que los escritores así como los lectores, en distintas disciplinas de CS\&S y de CB de los programas doctorales aquí en estudio, eligen recursos discursivos que se moldean en formatos similares, toda vez que se desea comunicar y difundir teorías y hallazgos empíricos.

En este análisis comparativo, especial mención merece el discurso de la disciplina de Física en el que no se detecta ningún género prototípico en este corpus. 
De los cinco géneros identificados en Física, ninguno es exclusivo. Todos ellos son compartidos con tres, cuatro, cinco o seis de estas disciplinas. Por su parte, el discurso de Lingüística y el de Química cuentan cada uno con un solo género exclusivo. En este mismo diagrama se aprecia que el discurso de Biotecnología (8), Literatura (7) e Historia (4) destaca por presentar una importante cantidad de géneros idiosincráticos, muchos de los cuales no registran una ocurrencia importante.

A continuación, en la Figura 4 se muestra, por medio del diagrama, la distribución jerárquica desde el discurso disciplinar de menor diversidad en géneros discursivos hasta aquél de mayor diversidad.

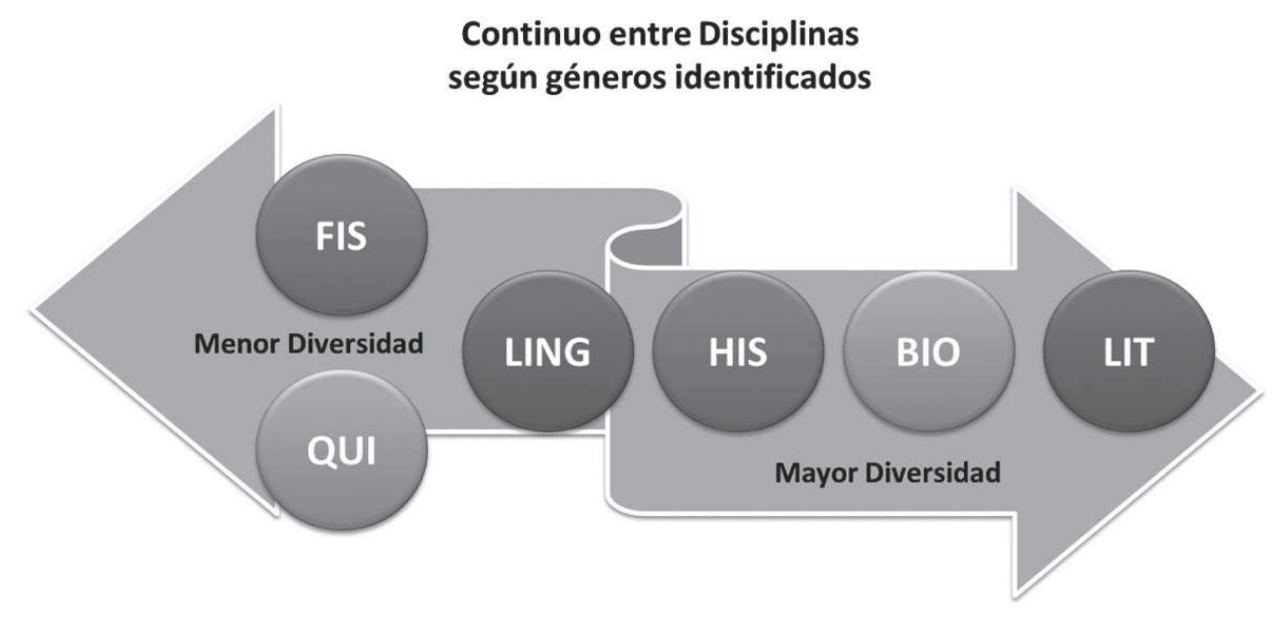

Figura 4. Disciplinas según diversidad de géneros.

Tal como se muestra, tanto el discurso de Física como el de Química revelan -comparativamente- menor diversidad de géneros (5), mientras que Literatura alcanza la mayor diversidad (15). Muy cerca, en términos numéricos de heterogeneidad de géneros, se encuentra el discurso de Biotecnología (14). En esta misma línea numérica, también aparece la disciplina de Historia con 13 géneros. En el centro de este continuum se ubica el discurso de Lingüística con un total de 7 géneros.

El análisis de este continuum de géneros por disciplina revela que, en cuanto a la variación genérica, no existe una clara separación entre el discurso de CS\&H y el de CB. Mientras el discurso de Lingüística se acerca en términos de relativa variación genérica al de Química y Física, el discurso de Biotecnología varía tan ampliamente como el discurso especializado de Historia y Literatura. Cabe recordar que el discurso disciplinar de Literatura es el único que muestra la singularidad de contar con dos macrogéneros: aquéllos denominados literarios y los no literarios. Esta particularidad sólo se identifica también en el discurso a nivel 
doctoral de la Historia, aunque muy posiblemente no bajo los mismos supuestos. En este sentido, es posible señalar que, en este caso, a los géneros que se incluyen en el macrogénero literario podría considerárselos como no especializados y más pertenecientes al ámbito general. En muchos casos, siguiendo a Parodi (2005), los textos de este macrogénero literario mostrarían rasgos prototípicos tales como -entre otros- uso de pronombres personales de primera persona singular y plural, de segunda persona singular y de tercera persona singular y plural; futuro perifrástico y pretérito imperfecto; adverbios de tiempo y de negación. Estos rasgos han sido asociados por oposición a una prosa no altamente compleja ni informacionalmente densa, característica de textos literarios orales y escritos y distantes de los textos técnicos especializados (Parodi, 2005).

\section{COMENTARIOS DE CIERRE}

Los hallazgos aportados por esta investigación dan cuenta de la identificación de 30 géneros del discurso a partir de un corpus de 3.147 textos, a través del discurso académico a nivel de doctorado en seis disciplinas: Biotecnología, Física, Historia, Lingüística, Literatura y Química. Estos resultados revelan una importante variedad genérica al interior del corpus; no obstante ello, la cuantificación de los textos pertenecientes a cada género muestra una alta predominancia de unos pocos géneros muy prototípicos del discurso académico y disciplinar en los estudios doctorales. Estos géneros más recurrentes y fundamentales son tres: Texto Disciplinar, Artículo de Investigación y Manual.

La distinción entre el discurso académico de las Ciencias Sociales y Humanidades y el de las Ciencias Básicas resulta particularmente significativa a nivel doctoral. Podría destacarse, por un lado, la mayor presencia del Manual en el discurso de CB, en contraste con la mayor ocurrencia del Texto Disciplinar en el discurso de CS\&H. Y, por otro, la tendencia a mayor variación genérica en el discurso de $\mathrm{CS} \& \mathrm{H}$, en comparación con una menor heterogeneidad de géneros identificados en el discurso de CB; aunque se detectan excepciones en uno y otro extremo. En este sentido, también se puede señalar una tendencia a contar con géneros exclusivos en CS\&H, mientras en el discurso de las CB se detectan menos géneros idiosincráticos.

Un hallazgo importante lo constituye la doble articulación de géneros detectada en Literatura e Historia. Desde el discurso de Literatura pueden distinguirse como de tipo literario y no literario. Lo que es común al discurso de ambas disciplinas es que unos abordan el estudio y análisis de los otros, revelando así la naturaleza preferentemente discursiva del tipo de investigación en Literatura e Historia, en comparación con una posible orientación más experimental en las otras disciplinas del Corpus PUCV-2010. 
Ahora bien, de modo más específico, cabe destacar que los dos géneros que presentan mayor ocurrencia en el discurso de las seis disciplinas estudiadas, según las cuantificaciones presentadas, y que están presentes en los seis corpus son el Texto Disciplinar y el Artículo de Investigación. El Manual se identifica en cinco de las seis disciplinas; no se reconoce únicamente en el discurso de Literatura. Este hallazgo, por un lado, podría indicar que, a pesar de la importante heterogeneidad genérica detectada a través del discurso de estas seis disciplinas doctorales, existiría una tendencia a la homogeneidad y coincidencia en los mecanismos discursivos de construcción de conocimientos especializados así como de su difusión. Esto querría decir que en estas seis disciplinas se detectaría una preferencia hacia organizaciones discursivas muy similares en disciplinas de Ciencias Sociales y Humanidades así como en otras de Ciencias Básicas. No obstante ello, por otro lado, también es factible pensar que estos géneros no serían tan homogéneos y podrían revelar características idiosincráticas y prototípicas de cada disciplina. En efecto, tanto Parodi (2008d) como Ibáñez (2008), a partir de un corpus de textos especializados en cuatro disciplinas, identificaron interesantes distinciones a través del discurso de algunas disciplinas de Ciencias Sociales y Humanidades y Ciencias Básicas en los géneros Manual y Texto Disciplinar, respectivamente. Estos hallazgos complementarios de investigaciones anteriores revelan que, aunque se trata de los mismos géneros, puesto que sus movidas retóricas nucleares se mantienen estables, se detectan otras movidas prototípicas en los géneros de unas y otras disciplinas.

Aunque en este caso no se ha realizado aún un estudio detallado de las características de unos y otros de los tres géneros identificados como transversales, y de alta ocurrencia en los seis corpus, es posible hipotetizar que sus rasgos podrían variar. Esto quiere decir que, a pesar de que son los mismos tres géneros (MA, AI y TD) que actúan como mecanismos discursivos fundamentales, ellos podrían mostrar particularidades específicas en cada disciplina. No es una novedad que un mismo género suele mostrar rasgos idiosincráticos como parte del discurso de una disciplina (Parodi, 2008d; Ibáñez, 2008; Martínez, 2012) o, incluso, a través de subdisciplinas (Burdiles, 2012). Asunto que, en este caso, obviamente debe corroborarse empíricamente en estudios posteriores.

Como es fácil inferir, los datos aportados en esta investigación ofrecen una diversa gama de potenciales proyecciones y abren escenarios novedosos a estudios complementarios. En lo más aplicado, estos hallazgos permiten pensar en la creación de programas de lectura en las disciplinas, tal como "Leer a través de las Disciplinas" (LAD); todo ello desde una perspectiva en que "la lingüística de corpus informe a la psicolingüística" (Parodi, 2011). 


\section{REFERENCIAS}

Araya, C. (2011). La presencia de esquemas argumentativos en los alegatos de clausura de fiscales y abogados defensores chilenos. Tesis para optar al grado de Doctor en Lingüística. Pontificia Universidad Católica de Valparaíso, Valparaíso, Chile.

Aravena, C. (2010). Artefactos visuales en textos académicos de Historia y Literatura a nivel de doctorado. Tesis para optar al grado de Magíster en Lingüística Aplicada. Pontificia Universidad Católica de Valparaíso, Chile.

Arnoux, E. (2002). La lectura y la escritura en la universidad. Buenos Aires: Eudeba.

Arnoux, E. (ed.) (2009). Escritura y producción de conocimiento en las carreras de postgrado. Buenos Aires: Arcos.

Bhatia, V. (1993). Analysing genre: Language use in professional settings. London: Longman.

Bhatia, V. (2004). Worlds of written discourse. A genre based view. Sydney: Continuun.

Bathia, V. (2012). Critical genre analysis. Conferencia Plenaria en el Congreso Internacional Rethinking Genre 20 Years Later, Ottawa, Canadá, 26-29 junio.

Bawarshi, A. y Reiff, M. (2010). Genre: An introduction to history, theory, research, and pedagogy (reference guides to rhetoric and composition). California: Parlor Press.

Bazerman, Ch. (1994). The informed writer: Using sources in the disciplines. New York: HMH.

Bazerman, Ch. (2012). A genre based Theory of Literate Action. Conferencia Plenaria en el Comgreso Internacional Rethinking Genre 20 Years Later, Ottawa, Canadá, 26-29 junio.

Bazerman, Ch. y Russell, D. (eds.) (1995). Landmark essays on writing across the curriculum. London: Routledge.

Bazerman, Ch. y Wiener, H. (2009). Writing skills handbook. California: Wadsworth Publishing.

Bazerman, Ch., Bonini, A. y Figueiredo, D. (eds.) (2009). Genre in a changing world. California: The Clearing House \& Parlor Press.

Bazerman, Ch., Krut, R., Lunsford, K., McLeod, S., Null, S., Rogers, P. y Stansell, A. (2009). Traditions of writing research. London: Routledge.

Bazerman, Ch., Dean, C., Early, J., Lunsford, K., Null, S., Rogers, P. y Stansell, A. (2012). International advances in writing research: cultures, places, measures. California: Parlor \& Clearing House Press.

Biber, D., Connor, U. y Upton, J. (2007). Discourse on the move. Using corpus analysis to describe discourse structure. Amsterdam: Benjamins. 
Burdiles, G. (2012). Descripción de la organización retórica del género Caso Clínico de la medicina a partir del Corpus CCM-2009. Tesis para optar al grado de Doctor en Lingüística. Pontificia Universidad Católica de Valparaíso, Valparaíso, Chile.

Castañeda. L. y Henao, J. (1995). La lectura en la universidad. Medellín: Universidad de Antioquía.

Castillo, C. (2012). La expresión de la subjetividad en la tesis de Licenciatura de Literatura y Lingüística: Una aproximación desde la teoría de la valoración. Seminario de Graduación para optar al grado de Licenciado en Lengua y Literatura Hispánica. Pontificia Universidad Católica de Valparaíso, Valparaíso, Chile.

Cubo de Severino, L., Lacon, N. y Puiatti, H. (eds.) (2012). Escribir una Tesis. Manual de estrategias de producción. Córdoba: Comunicarte.

Devitt, A. (2008). Writing genres (rhetorical philosophy and theory). California: Parlor Press.

Farlora, M. (2011). Descripción funcional del género académico didáctico con función evaluativa prueba tipo ensayo en el área humanidades: Diferencias disciplinares. Tesis para optar al Grado de Doctor en Lingüística. Pontificia Universidad Católica de Valparaíso, Valparaíso, Chile.

Faune, C. (2012). Caracterización de trabajos finales de grado de Licenciatura en Filosofía, Lingüística, Literatura y Psicología de la PUCV. Seminario de Graduación para optar al grado de Licenciado en Lengua y Literatura Hispánica. Pontificia Universidad Católica de Valparaíso, Valparaíso, Chile.

Freedman, A. y Medway, P. (eds.) (1994). Genre and the new rhetoric. London: Taylor \& Francis.

González, C. (2011). La formulación de los objetivos en artículos de investigación científica en cuatro disciplinas: historia, lingüística, literatura y biología. Linguagem em (Dis)curso, 11(2), 401-429.

Grassi, G. (2012). Análisis retórico-discursivo de la Conclusión en trabajos finales de Grado de Licenciatura en Lingüística, Literatura, Filosofía y Psicología. Seminario de Graduación para optar al grado de Licenciado en Lengua y Literatura Hispánica. Pontificia Universidad Católica de Valparaíso, Valparaíso, Chile.

Hyland, K. (2012). The ESP Version: Genre, community and identity. Conferencia Plenaria en el Congreso Internacional Rethinking Genre 20 Years Later, Ottawa, Canadá, 26-29 junio.

Hyon, S. (1996). Genre in three traditions: Implications for ESL. TESOL Quarterly, 30(4), 693-722.

Ibáñez, R. (2008). El Texto Disciplinar y el acceso al conocimiento desde el análisis del género: ¿Regulación del conocimiento o persuasión? En G. Parodi (ed.), 
Géneros académicos y géneros profesionales. Accesos discursivos para saber y hacer (pp. 219-246). Valparaíso: EUV.

Jarpa, M. (2012). Macrogénero académico evaluativo: Descripción retórica-estructural en la comunidad de aprendizaje de Postgrado en Biotecnología. Tesis para optar al Grado de Doctor en Lingüística. Pontificia Universidad Católica de Valparaíso, Valparaíso Chile.

Lobos, I. (2012). Caracterización del posicionamiento en Conclusiones de Tesis de Licenciatura, Magíster y Doctorado en Filosofía y Lingüística. Seminario de graduación para optar al grado de Licenciado en Lengua y Literatura Hispánica. Pontificia Universidad Católica de Valparaíso, Valparaíso, Chile.

Marinkovich, J. (ed.) (2012). La escritura como proceso: Investigación y práctica en el aula. Madrid: Editorial Académica Española.

Marinkovich, J., Morán, P. y Benítez, R. (1997). La enseñanza de la escritura en el aula. Una metodología interdisciplinaria. Valparaíso. Imprenta Carroza.

Marinkovich, J., Peronard, M. y Parodi, G. (2006). LECTES: Programa de lectura y escritura. Valparaíso: EUV.

Marinkovich, J., Velásquez, M., Salazar, J. y Córdova, A. (2009). Aprendiendo a escribir en las disciplinas: Articulación entre el curriculum escolar y el universitario. Valparaíso: EUV.

Martin, J. (2012). Genre, identity and restorative justice: Rule and Role in Youth Justice Conference. Conferencia Plenaria en el Congreso Internacional Rethinking Genre 20 Years Later, Ottawa, Canadá, 26-29 junio.

Martin, J. y Rose, D. (2008). Genre relations. Mapping culture. London: Equinox. Martínez, J.D. (2012). Descripción y variación retórico-funcional del género Tesis Doctoral: Un análisis desde dos disciplinas y dos comunidades discursivas a partir del Corpus Te DiCE-2010. Tesis para optar al Grado de Doctor en Lingüística. Pontificia Universidad Católica de Valparaíso, Chile.

Miller, C. (1984). Genre as social action. Quarterly Journal of Speech, 70, 151-167.

Miller, C. (2012). Do genres evolve? Conferencia Plenaria en el Congreso Internacional Rethinking Genre 20 Years Later, Ottawa, Canadá, 26-29 junio.

Parodi, G. (2003). Relaciones entre lectura y escritura: Una perspectiva cognitiva discursiva. Bases teóricas y antecedentes empiricos. Valparaíso: EUV.

Parodi, G. (2004). Textos de especialidad y comunidades discursivas técnico-profesionales: Una aproximación basada en corpus computarizado. Revista Estudios Filológicos, 39, 7-36.

Parodi, G. (2005). Lingüística de corpus y análisis multidimensional: Exploración de la variación en el Corpus PUCV-2003. En G. Parodi (ed.), Discurso especializado e instituciones formadoras (pp. 83-126). Valparaíso: EUV.

Parodi, G. (Ed.) (2008a). Géneros académicos y profesionales. Accesos discursivos para saber y hacer. Valparaíso: EUV. 
Parodi, G. (2008b). Géneros académicos universitarios: Develando un discurso de naturaleza mixta. En G. Parodi (ed.), Géneros académicos y géneros profesionales. Accesos discursivos para saber y hacer (pp. 97-116). Valparaíso: EUV.

Parodi, G. (2008c). Géneros del discurso escrito: Hacia una concepción integral desde una perspectiva sociocognitiva. En G. Parodi (ed.), Géneros académicos y géneros profesionales. Accesos discursivos para saber y hacer (pp. 17-37). Valparaíso: EUV.

Parodi, G. (2008d). El género Manual y su organización retórica en cuatro disciplinas científicas: Entre la abstracción y la concreción. En G. Parodi (ed.), Géneros académicos y géneros profesionales. Accesos discursivos para saber y hacer (pp. 199-218). Valparaíso: EUV.

Parodi, G. (2009). El corpus académico y profesional del Español PUCV-2006: Semejanzas y diferencias entre los géneros académicos y profesionales. Revista Estudios Filológicos, 44, 123-147.

Parodi, G. (ed.) (2010a). Alfabetización académica y disciplinar. Leer y escribir en las disciplinas. Santiago de Chile: Ariel.

Parodi, G. (2010b). Multisemiosis y lingüística de corpus: Artefactos (multi)semióticos en los textos de seis disciplinas en el Corpus PUCV-2010. Revista de Lingüistica Teórica y Aplicada, RLA, 48, 233-270.

Parodi, G. (2011). La Teoría de la Comunicabilidad: Notas para una concepción integral de la comprensión de textos escritos. Revista Signos. Estudios de Lingüistica, 44 (76), 145-167.

Parodi, G. (2012). Programa Leer, hablar y escribir a través del discurso del Derecho. Facultad de Derecho, Pontificia Universidad Católica de Valparaíso, Valparaíso, Chile.

Parodi, G. y Gramajo, A. (2003). Los tipos textuales del Corpus PUCV-2003: Una aproximación multiniveles. Revista Signos. Estudios de Lingüistica, 36(54), 207-223.

Parodi, G., Venegas, R., Ibáñez, R. y Gutiérrez. R.M. (2008). Géneros del discurso en el Corpus PUCV-2006: Criterios, definiciones y ejemplos. En G. Parodi (ed.), Géneros académicos y profesionales: accesos discursivos para saber y hacer (pp. 39-74) .Valparaíso: EUV.

Peronard, M., Gómez, L., Parodi, G., Núñez, P. y González, J. (1998). Programa LyC: Leer y comprender. Santiago de Chile: Editorial Andrés Bello.

PLEA (2012). Programa de Lectura y Escrituras Académicas. Pontificia Universidad Católica de Chile, Chile.

Resnik, G. y Valente, E. (2009). La lectura y la escritura en el trabajo de taller: Aspectos metodológicos. Buenos Aires: Universidad Nacional General Sarmiento.

Rodríguez, V. (2012). Caracterización retórico-discursiva del apartado Introducción en Tesis de Licenciatura en Literatura, Lingüística, Filosofía y Psicología. Seminario de graduación para optar al grado de Licenciado en Lengua y Li- 
teratura Hispánica. Pontificia Universidad Católica de Valparaíso, Valparaíso, Chile.

Russell, D. (2002). Writing in the academic disciplines: A curricular history. Carbondale: Southern Illinois University Press.

Swales, J. (1990). Genre analysis. English in academic and research settings. Cambridge: Cambridge University Press.

Swales, J. (2004). Research genres: Exploration and applications. Cambridge: Cambridge University Press.

Swales, J. (2012). Texts and commentaries: Toward a reception study of "genre tradition" (Hyon, 1996). Conferencia Plenaria en el Congreso Internacional Rethinking Genre 20 Years Later, Ottawa, Canadá, 26-29 junio.

Tognini-Bonelli, E. (2001). Corpus linguistics at work. Amsterdam: Benjamins. Zamora, S. (2012). Modos de organización textual en trabajos finales de grado en el nivel de Licenciatura: Caracterización a partir de densidades. Seminario de Graduación para optar al grado de Licenciado en Lengua y Literatura Hispánica. Pontificia Universidad Católica de Valparaíso, Valparaíso, Chile. 